\title{
Genome-wide identification, structural and gene expression analysis of the bZIP transcription factor family in sweet potato wild relative Ipomoea trifida
}

Zhengmei Yang ${ }^{1,2+}$, Jian Sun ${ }^{1,2+}$, Yao Chen ${ }^{1,2}$, Panpan Zhu ${ }^{3}$, Lei Zhang ${ }^{1,2}$, Shaoyuan $\mathrm{Wu}^{1,2}$, Daifu Ma ${ }^{4}$, Qinghe Cao ${ }^{4}$, Zongyun $\mathrm{Li}^{1,2^{*}}$ and Tao $\mathrm{Xu}^{1,2^{*}}$ (D)

\begin{abstract}
Background: The basic leucine zipper (bZIP) transcription factor is one of the most abundant and conserved transcription factor families. In addition to being involved in growth and development, bZIP transcription factors also play an important role in plant adaption to abiotic stresses.

Results: A total of 41 bZIP genes that encode 66 proteins were identified in Ipomoea trifida. They were distributed on 14 chromosomes of Ipomoea trifida. Segmental and tandem duplication analysis showed that segmental duplication played an important role in the ItfbZIP gene amplification. ItfbZIPs were divided into ten groups (A, B, C, D, E, F, G, H, I and S groups) according to their phylogenetic relationships with Solanum lycopersicum and Arabidopsis thaliana. The regularity of the exon/intron numbers and distributions is consistent with the group classification in evolutionary tree. Prediction of the cis-acting elements found that promoter regions of ItfbZIPs harbored several stress responsive cis-acting elements. Protein three-dimensional structural analysis indicated that ItfbZIP proteins mainly consisted of a-helices and random coils. The gene expression pattern from transcriptome data and GRT-PCR analysis showed that ItfbZIP genes expressed with a tissue-specific manner and differently expressed under various abiotic stresses, suggesting that the ItfbZIPs were involved in stress response and adaption in Ipomoea trifida.
\end{abstract}

Conclusions: Genome-wide identification, gene structure, phylogeny and expression analysis of bZIP gene in Ipomoea trifida supplied a solid theoretical foundation for the functional study of bZIP gene family and further facilitated the molecular breeding of sweet potato.

Keywords: Ipomoea trifida, Sweet potato, bZIP transcription factor, Phylogenetic analysis, Gene expression

\section{Background}

Transcription factors (TFs) are active proteins that recognize and bind to specific sites on a promoter to activate or inhibit gene expression $[1,2]$. The basic leucine zipper (bZIP) TFs is one of the most diverse families of TFs $[3,4]$. Structurally, this family contains a highly conserved bZIP domain (60-80 amino acids long) which is divided into two parts: the basic region and the leucine zipper region [5]. The basic region, which is the most

\footnotetext{
* Correspondence: zongyunli@jsnu.edu.cn; xutao_yr@126.com

${ }^{\dagger}$ Zhengmei Yang and Jian Sun contributed equally to this work.

'Institute of Integrative Plant Biology, School of Life Sciences, Jiangsu Normal

University, Xuzhou 221116, Jiangsu Province, China

Full list of author information is available at the end of the article
}

conserved core part of the bZIP TF, consists of approximately 16 amino acid residues. This region contains an invariant motif $\mathrm{N}-\mathrm{X} 7-\mathrm{R} / \mathrm{K}-\mathrm{X} 9$, which is mainly involved in nuclear localization and target DNA binding function. Meanwhile, the leucine zipper region is less conserved and composed of heptad repeats of Leu or other bulky hydrophobic amino acids (Ile, Val, Phe or Met) positioning exactly the nine amino acids towards the $C$ terminus $[1$, 2]. Through the interaction of the hydrophobic amino acids in the helical region, the two subunits are tightly bound together to form a coiled-coil dimer structure. This structure affects the binding characteristics, expression diversity and gene regulation of the target gene. 
To date, the bZIP gene family has been widely identified by genome-wide analyses in various plants, such as $75 \mathrm{Atb}$ ZIPs identified in Arabidopsis thaliana [6], 89 OsbZIPs in Oryza sativa [3], 131 GmbZIPs in Glycine max [7], 92 SbbZIPs in Sorghum bicolor [8], 125 ZybZIPs in Zea mays [9], 55 VvbZIPs in Vitis vinifera [10], 64 CsbZIPs in Cucumis sativus [11], 77 MebZIPs in Maninot esculenta crantz [12], 112 MdbZIPs in Malus domestica Borkh [13] and 247 BrbZIPs in Brassica napus [14]. bZIP TFs are involved in the regulation of seed development $[15,16]$, cell elongation [17, 18], vascular development [17], flower development [19-22], somatic embryogenesis [23] and nitrogen/carbon and energy metabolism [24-26]. In addition of the essential functions of bZIPs in plant growth and development, $b Z I P s$ play important roles in plants under abiotic stress conditions. AtbZIP17, AtbZIP24, OsbZIP12, OsbZIP72, OsABF1, ThbZIP1, GmbZIP44, GmbZIP62 and GmbZIP78 directly or indirectly positively regulate the salt stress adaption in plants [7, 27-32]. OsbZIP52/RISBZ5, OsbZIP16, OsbZIP23, OsbZIP45, AREB1, AREB2, ABF3 and ThbZIP1 are involved in drought tolerance [33-36]. LIP19 is a Fos-like molecular switch in cold signalling [37]. OsbZIP52/RISBZ5 negatively regulates cold stress response [33]. OsbZIP72 is a positive regulator of ABA response [29], and overexpression of GmbZIP44, GmbZIP62 and GmbZIP78 attenuates ABA sensitivity [7]. However, the $b Z I P$ gene family has not been characterized in sweet potato.

Sweet potato (Ipomoea batatas) of the family Convolvulaceae is an annual or perennial herb with an extremely high economic value. To date, sweet potato is the seventh largest crop in the world. It is an important food crop, forage crop and a new type of bio-energy crop in China. Sweet potato is a hexaploid cultivar with 90 chromosomes. Due to its large genome and high genetic heterogeneity, the whole genome sequencing and assembly as well as the other related genomics research is very complicated. However, diploid Ipomoea trifida G. Don $(2 \mathrm{n}=2 \mathrm{x}=30)$ which belongs to Batata section B of the genera Ipomoea, is considered as an ancestral species of hexaploid cultivated sweet potato [38-40]. The diploid Ipomoea trifida is an ideal model species for studying self-incompatibility, genetic mapping, physical mapping, sweet potato breeding, sweet potato transgenic system construction and whole genome sequencing due to its small size, low ploidy, small chromosome number and simple genetic manipulation. In 2017 , the genome data of diploid sweet potato variety Ipomoea trifida were released (http://sweetpotato.plantbiol ogy.msu. edu/), making it possible to identify and analyse important gene families at the whole genome level in Ipomoea trifida [41].

In this study, bZIP gene family members were identified in Ipomoea trifida. Using various bioinformatics tools, we performed ItfbZIP subfamily classification, gene intron/ exon distribution analysis, conserved domain and three-dimensional structural homology modeling prediction. And the ItfbZIP gene expression profiles generated from RNA-seq were confirmed by quantitative RT-PCR in different plant tissues under various abiotic stresses. This study could be helpful for further functional study of $b Z I P$ genes and molecular breeding of sweet potato.

\section{Results \\ Identification and designation of bZIP TFs in the Ipomoea trifida genome}

A total of 66 ItfbZIP proteins encoded by 41 ItfbZIP genes were identified. They all include at least one bZIP domain (bZIP_1: PF00170.20, bZIP_2: PF07716.14 or bZIP_Maf: PF03131). The ItfbZIP genes were numbered according to its position on the chromosome as ItfbZIP1 ItfbZIP41. Different transcripts encoded by the same gene were given the similar names. For instance, 3 transcripts of ItfbZIP9 gene are ItfbZIP9.1, ItfbZIP9.2 and ItfbZIP9.3 (Additional file 1: Table S1). At the same time, ItfbZIP protein size (aa), MW, pI, subcellular location and phosphorylation site were analyzed (Table 1). The length of ItfbZIP proteins ranges from 158 AA (ItfbZIP41.1) to 754AA (ItfbZIP40), MW varies from 17,227.12 (ItfbZIP41.1) to 81,305.95 (ItfbZIP40) Da and pI distributes from 5.61 (ItfbZIP14) to 9.69 (ItfbZIP41.1). They are all predicted to be located in the nucleus. We used the online tool P3DB to predict the phosphorylation sites of the ItfbZIPs. As listed in Table 1, the ItfbZIPs contain 4 to 28 phosphorylatio sites, wherein the maximum number of phosphorylation sites is 28 for ItfbZIP34.1 and ItfbZIP34.2. The minimum number of phosphorylation sites is 4 for ItfbZIP25.2 and ItfbZIP27.2. About 60\% of the ItfbZIPs contain 10 or more phosphorylation sites. Up to $80 \%$ of the ItfbZIPs contain more Ser phosphorylation sites than Thr phosphorylation sites.

\section{Chromosome localization and duplication of the ItfbZIP gene family}

The ItfbZIP genes were mapped on 15 chromosomes. As shown in Fig. 1, nine ItfbZIP genes on Chr 9; five ItfbZIP genes on Chr 5; four on Chr 10; three on Chr 1, Chr 3, Chr 14 and Chr 15; two on Chr 4, Chr 7, Chr 8 and Chr 12; only one on Chr 2, Chr 6 and Chr 11; and no ItfbZIP gene on Chr 13, indicating that the distribution of ItfbZIP genes is disproportionate on chromosomes.

The amplification of gene family in plant evolution is mainly carried out by genome duplication [42, 43]. To investigate possible relationships among the ItfbZIP genes and potential gene duplication type, we performed collinear analysis. (Fig. 1 and Additional file 1: Table S1). According to Holub's description [44], a chromosomal region within $200 \mathrm{~kb}$ containing two or more genes was defined as a tandem duplication event. The results indicated that the ItfbZIP gene has no tandem duplication. 
Table 1 Characteristics of ItfbZIPs in I. trifida

\begin{tabular}{|c|c|c|c|c|c|c|c|c|}
\hline \multirow{2}{*}{$\begin{array}{l}\text { Gene } \\
\text { name }\end{array}$} & \multirow[t]{2}{*}{ Gene ID } & \multirow{2}{*}{$\begin{array}{l}\text { Amino } \\
\text { acids }\end{array}$} & \multirow[t]{2}{*}{ MW(Da) } & \multirow[t]{2}{*}{$\mathrm{Pl}$} & \multirow{2}{*}{$\begin{array}{l}\text { Subcellular } \\
\text { location }\end{array}$} & \multicolumn{3}{|c|}{ No. Of phosphorylation site } \\
\hline & & & & & & Ser site & Thr site & Total \\
\hline $\mid t f b Z I P 1$ & itf01g16700.t1 & 159 & $17,242.26$ & 9.69 & Nucleus. & 3 & 2 & 5 \\
\hline ItfbZIP2 & itf01g24710.t1 & 468 & $51,081.9$ & 7.23 & Nucleus. & 10 & 4 & 14 \\
\hline ItfbZIP3.1 & itf01g27620.t1 & 432 & $46,967.07$ & 6.22 & Nucleus. & 7 & 7 & 11 \\
\hline ItfbZIP3.2 & itf01g27620.t2 & 317 & $33,951.71$ & 5.63 & Nucleus. & 13 & 7 & 20 \\
\hline ItfbZIP4 & itf02g14930.t1 & 322 & $35,055.77$ & 5.66 & Nucleus. & 7 & 4 & 11 \\
\hline ItfbZIP5 & itf03g14110.t1 & 340 & $37,151.20$ & 6.2 & Nucleus. & 9 & 7 & 16 \\
\hline ItfbZIPG & itf03g16790.t1 & 292 & $32,438.48$ & 6.19 & Nucleus. & 4 & 1 & 5 \\
\hline ItfbZIP7.1 & itf03g29500.t1 & 372 & $42,071.81$ & 6.43 & Nucleus. & 8 & 5 & 13 \\
\hline ItfbZIP7.2 & itf03g29500.t2 & 324 & $36,655.73$ & 6.35 & Nucleus. & 5 & 4 & 9 \\
\hline ItfbZIP8 & itf04g28510.t1 & 173 & $20,455.39$ & 6.92 & Nucleus. & 2 & 5 & 7 \\
\hline ItfbZIP9.1 & itf04g33190.t1 & 328 & $36,566.17$ & 7.07 & Nucleus. & 5 & 5 & 10 \\
\hline ItfbZIP9.2 & itf04g33190.t4 & 285 & $31,492.39$ & 5.74 & Nucleus. & 5 & 3 & 8 \\
\hline ItfbZIP9.3 & itf04g33190.t6 & 295 & $32,815.01$ & 9.2 & Nucleus. & 3 & 2 & 5 \\
\hline ItfbZIP10.1 & itf05g21690.t1 & 383 & $43,053.99$ & 6.28 & Nucleus. & 5 & 5 & 10 \\
\hline ItfbZIP10.2 & itf05g21690.t2 & 373 & $42,042.89$ & 6.54 & Nucleus. & 7 & 4 & 11 \\
\hline ItfbZIP10.3 & itf05g21690.t3 & 335 & $37,582.79$ & 6.12 & Nucleus. & 4 & 5 & 9 \\
\hline$|t f b Z| P 11$ & itf05g23650.t1 & 227 & $24,148.57$ & 9.62 & Nucleus. & 4 & 3 & 7 \\
\hline ItfbZIP12 & itf05g12890.t1 & 375 & $42,461.33$ & 5.53 & Nucleus. & 11 & 2 & 13 \\
\hline ItfbZIP13 & itf05g01550.t1 & 335 & $37,844.91$ & 5.91 & Nucleus. & 11 & 2 & 13 \\
\hline ItfbZIP14 & itf05g02250.t1 & 347 & $37,706.85$ & 5.61 & Nucleus. & 12 & 8 & 20 \\
\hline ItfbZIP15.1 & itf06g21610.t1 & 453 & $49,470.31$ & 7.82 & Nucleus. & 7 & 8 & 15 \\
\hline ItfbZIP15.2 & itf06g21610.t2 & 374 & $40,435.16$ & 8.51 & Nucleus. & 6 & 7 & 13 \\
\hline ItfbZIP16 & itf07g01470.t1 & 327 & $36,572.2$ & 8.66 & Nucleus. & 3 & 5 & 8 \\
\hline ItfbZIP17.1 & itf07g00390.t1 & 482 & $53,284.76$ & 6.22 & Nucleus. & 7 & 6 & 13 \\
\hline ItfbZIP17.2 & itf07g00390.t2 & 501 & $55,317.93$ & 6.47 & Nucleus. & 4 & 3 & 7 \\
\hline ItfbZIP17.3 & itf07g00390.t3 & 414 & $45,910.63$ & 7.22 & Nucleus. & 4 & 2 & 6 \\
\hline ItfbZIP17.4 & itf07g00390.t4 & 404 & $44,649.24$ & 6.64 & Nucleus. & 5 & 3 & 8 \\
\hline ItfbZIP18 & itf08g03360.t1 & 455 & $49,697.54$ & 6.1 & Nucleus. & 13 & 4 & 17 \\
\hline ItfbZIP19.1 & itf08g03030.t1 & 178 & $20,232.97$ & 10.3 & Nucleus. & 1 & 5 & 6 \\
\hline ItfbZIP19.2 & itf08g03030.t2 & 181 & $20,703.43$ & 10.3 & Nucleus. & 1 & 4 & 5 \\
\hline ItfbZIP19.3 & itf08g03030.t4 & 166 & $18,873.5$ & 10.52 & Nucleus. & 2 & 3 & 5 \\
\hline ItfbZIP2O & itf09g13580.t1 & 540 & 58,567 & 6.09 & Nucleus. & 6 & 5 & 11 \\
\hline ItfbZIP21 & itf09g11330.t1 & 469 & $51,205.22$ & 7.86 & Nucleus. & 7 & 5 & 12 \\
\hline ItfbZIP22.1 & itf09g10570.t1 & 331 & $36,463.9$ & 6.41 & Nucleus. & 3 & 8 & 11 \\
\hline ItfbZIP22.2 & itf09g10570.t2 & 316 & $34,757.9$ & 6.02 & Nucleus. & 3 & 7 & 10 \\
\hline ItfbZIP23.1 & itf09g04120.t1 & 369 & $41,756.38$ & 8.46 & Nucleus. & 11 & 4 & 15 \\
\hline ItfbZIP23.2 & itf09g04120.t2 & 322 & $36,206.8$ & 9.2 & Nucleus. & 8 & 5 & 13 \\
\hline ItfbZIP24.1 & itf09g00830.t1 & 593 & $64,463.21$ & 6.8 & Nucleus. & 15 & 4 & 19 \\
\hline ItfbZIP24.2 & itf09g00830.t2 & 495 & $53,646.59$ & 6.62 & Nucleus. & 13 & 5 & 18 \\
\hline ItfbZIP25.1 & itf09g13780.t1 & 286 & $31,976.66$ & 5.94 & Nucleus. & 4 & 3 & 7 \\
\hline ItfbZIP25.2 & itf09g13780.t2 & 235 & $26,356.41$ & 6.33 & Nucleus. & 2 & 2 & 4 \\
\hline ItfbZIP25.3 & itf09g13780.t3 & 445 & $49,462.53$ & 6.42 & Nucleus. & 8 & 5 & 13 \\
\hline ItfbZIP25.4 & itf09g13780.t4 & 394 & $43,842.28$ & 6.76 & Nucleus. & 7 & 7 & 14 \\
\hline
\end{tabular}


Table 1 Characteristics of ItfbZIPs in I. trifida (Continued)

\begin{tabular}{|c|c|c|c|c|c|c|c|c|}
\hline \multirow{2}{*}{$\begin{array}{l}\text { Gene } \\
\text { name }\end{array}$} & \multirow[t]{2}{*}{ Gene ID } & \multirow{2}{*}{$\begin{array}{l}\text { Amino } \\
\text { acids }\end{array}$} & \multirow[t]{2}{*}{ MW(Da) } & \multirow[t]{2}{*}{$\mathrm{Pl}$} & \multirow{2}{*}{$\begin{array}{l}\text { Subcellular } \\
\text { location }\end{array}$} & \multicolumn{3}{|c|}{ No. Of phosphorylation site } \\
\hline & & & & & & Ser site & Thr site & Total \\
\hline ItfbZIP25.5 & itf09g13780.t7 & 429 & $47,877.84$ & 7.29 & Nucleus. & 7 & 6 & 13 \\
\hline ItfbZIP26 & itf09g15920.t1 & 176 & $19,677.33$ & 9.21 & Nucleus. & 3 & 2 & 5 \\
\hline ItfbZIP27.1 & itf09g23000.t1 & 205 & $22,960.18$ & 5.49 & Nucleus. & 5 & 6 & 11 \\
\hline ItfbZIP27.2 & itf09g23000.t2 & 182 & $20,462.37$ & 5.79 & Nucleus. & 2 & 2 & 4 \\
\hline ItfbZIP28.1 & itf09g23040.t1 & 438 & $46,873.95$ & 5.62 & Nucleus. & 9 & 4 & 13 \\
\hline ItfbZIP28.2 & itf09g23040.t2 & 433 & $46,272.23$ & 5.52 & Nucleus. & 12 & 5 & 17 \\
\hline ItfbZIP29 & itf10g21980.t1 & 359 & $41,241.48$ & 7.29 & Nucleus. & 10 & 3 & 13 \\
\hline ItfbZIP30 & itf10g22380.t1 & 290 & $30,693.36$ & 8.53 & Nucleus. & 4 & 5 & 9 \\
\hline$|t f b Z| P 31$ & itf10g25610.t1 & 289 & $31,158.79$ & 6.19 & Nucleus. & 3 & 4 & 7 \\
\hline ItfbZIP32 & itf10g09640.t1 & 436 & $47,072.45$ & 6.09 & Nucleus. & 11 & 6 & 17 \\
\hline ItfbZIP33 & itf1 1g03980.t1 & 312 & $34,536.45$ & 5.55 & Nucleus. & 8 & 1 & 9 \\
\hline ItfbZIP34.1 & itf12g08780.t1 & 549 & $56,965.27$ & 6.2 & Nucleus. & 18 & 10 & 28 \\
\hline ItfbZIP34.2 & itf12g08780.t2 & 553 & $57,378.75$ & 6.33 & Nucleus. & 16 & 12 & 28 \\
\hline ItfbZIP35 & itf12g23960.t1 & 349 & $38,224.45$ & 5.75 & Nucleus. & 12 & 7 & 19 \\
\hline ItfbZIP36 & itf14g02130.t1 & 479 & $52,507.02$ & 5.78 & Nucleus. & 8 & 4 & 12 \\
\hline ItfbZIP37.1 & itf14g09670.t1 & 328 & $36,764.3$ & 8.64 & Nucleus. & 4 & 5 & 9 \\
\hline ItfbZIP37.2 & itf14g09670.t3 & 294 & $32,965.99$ & 9.29 & Nucleus. & 2 & 3 & 5 \\
\hline ItfbZIP38.1 & itf14g12010.t1 & 512 & $56,187.72$ & 6.47 & Nucleus. & 8 & 4 & 12 \\
\hline ItfbZIP38.2 & itf14g12010.t2 & 508 & $55,855.41$ & 6.47 & Nucleus. & 8 & 5 & 13 \\
\hline ItfbZIP39 & itf15g02970.t1 & 546 & $60,189.56$ & 6.82 & Nucleus. & 12 & 4 & 16 \\
\hline ItfbZIP40 & itf15g04720.t1 & 754 & $81,305.95$ & 6.25 & Nucleus. & 13 & 5 & 18 \\
\hline ItfbZIP41.1 & itf15g19800.t1 & 158 & $17,227.12$ & 9.69 & Nucleus. & 3 & 3 & 6 \\
\hline ItfbZIP41.2 & itf15g19800.t2 & 184 & $20,395.82$ & 7.03 & Nucleus. & 3 & 3 & 6 \\
\hline
\end{tabular}

Segmental duplications were identified using the BLASTP and MCScanX methods and 13 segmental duplications were found. As followers: ItfbZIP1-ItfbZIP41, ItfbZIP2-ItfbZIP21, ItfbZIP5-ItfbZIP14, ItfbZIP5-ItfbZIP35, ItfbZIP9-ItfbZIP16, ItfbZIP9-ItfbZIP37, ItfbZIP14-ItfbZIP5, ItfbZIP15-ItfbZIP18, ItfbZIP15-ItfbZIP24, ItfbZIP16-ItfbZIP37, ItfbZIP17-ItfbZIP38, ItfbZIP18-ItfbZIP24, ItfbZIP23-ItfbZIP29.

\section{Distribution of bZIP TFs in eukaryotes}

To understand the evolutionary relationship of bZIP TFs among different eukaryotes, we performed phylogenetic tree construction on 28 species (including fungi, metazoa and plants) and annotated every species with the number of bZIPTFs identified in previous literatures (Fig. 2 and Additional file 2: Table S2). The figure shows only a few bZIP homologs exist in Saccharomyces cerevisiae (17) and Ustilaginoidea virens (28). However, plants have a large number of $b Z I P$ genes. The number of bZIPTFs in monocotyledonous plants ranges from 89 to 98 with exceptions of 125 for Zea mays and 141 for Hordeum vulgare, which may be due to the common ancestor and similar complete genome duplication of the gramineous plants [45]. The range of bZIPTFs in dicotyledonous plants is 41 to 247 , wherein the maximum number of bZIPTFs in tetraploid Brassica napus is 247, probably due to the large number of gene duplications. Although Ipomoea trifida and Solanum lycopersicum belong to Solanales, the number of ItfbZIP gene (41) is 28 less than that of SlbZIPs (69), indicating that the bZIP gene number of Ipomoea trifida has been reduced during evolution. In general, the numbers of bZIPs in Homo sapiens and higher plants are more than that in fungus, lower animals and plants, which may be closely related to the ability to regulate physiological responses to environmental stimuli in eukaryotic species.

\section{Phylogenetic relationship of bZIP proteins in Arabidopsis thaliana, Solanum lycopersicum and Ipomoea trifida}

To study the evolutionary relationship of bZIPs among Ipomoea trifida, Arabidopsis thaliana and Solanum lycopersicum, we established phylogenetic tree of bZIPs of these three species using MEGA7 by Maximum Likelihood method with bootstrap analysis (1000 replicates) (Fig. 3 and Additional file 3: Table S3). The phylogenetic tree consists of 75 Arabidopsis thaliana, 69 Solanum 


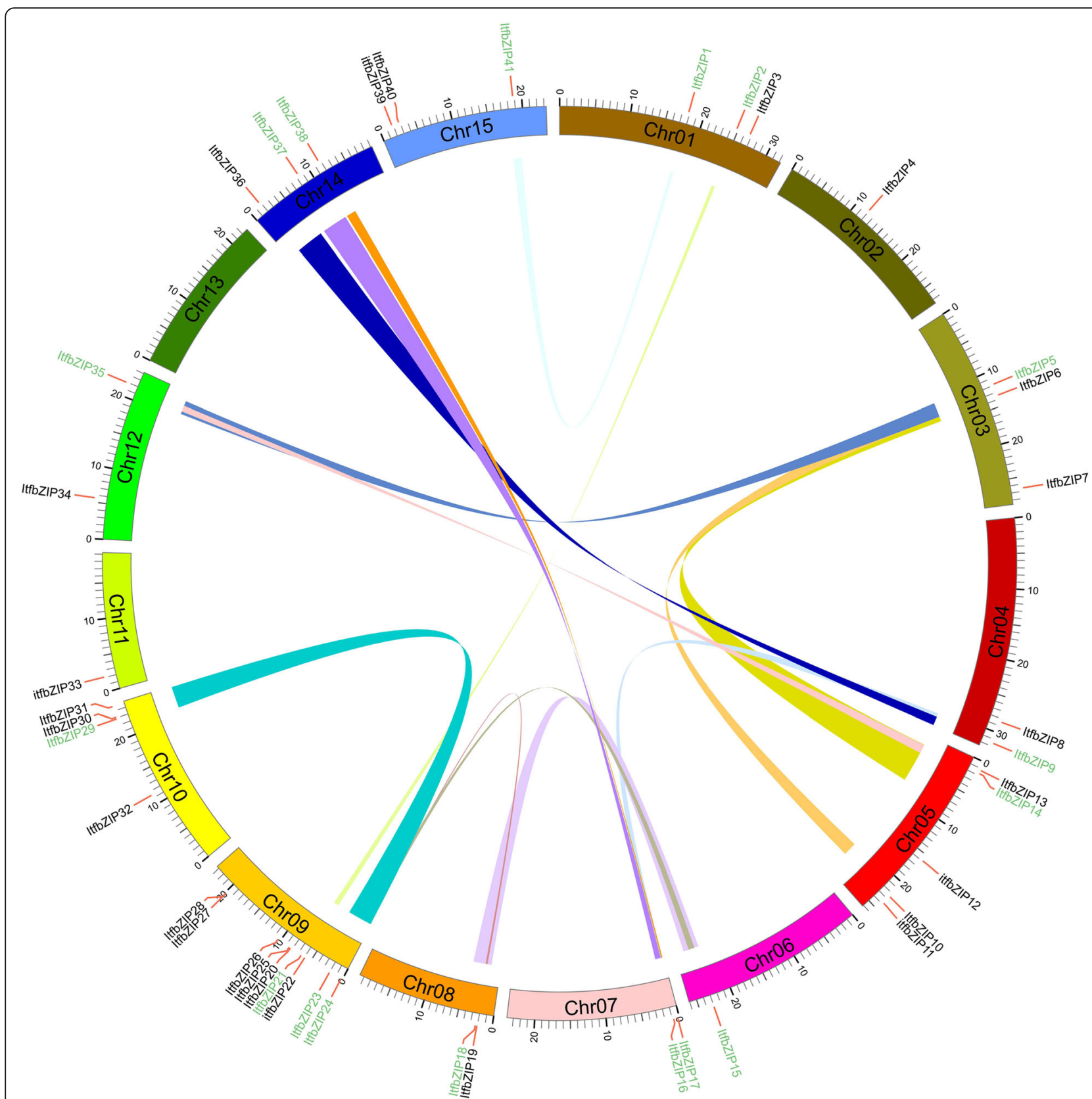

Fig. 1 Distribution and segmental duplication of ItfbZIP genes in Ipomoea trifida Chromosomes. 41 ItfbZIP genes were mapped to the 14 chromosomes. Different colored lines indicated the segmental duplication. The red line next to the name indicates the gene cluster on each chromosome. Gene names with collinearity are colored in green, and no collinear gene names are colored in black. The chromosomal location and segmental duplication of the ItfbZIP genes are in Table S1

lycopersicum and 66 Ipomoea trifida bZIP protein sequences. The 75 bZIP TFs identified in Arabidopsis thaliana were divided into 10 subgroups of A-I and S [6]. The specific distribution of the ItfbZIP proteins was as follows: A (8), B (1), C (6), D (22), E (7), F (2), G(5), H(3), I(11) and S(1). Among the 66 ItfbZIPs, $18 \%$ of the ItfbZIPs is close related AtbZIPs, and 72\% of the ItfbZIPs is close related SLbZIPs, which is consistent with that Ipomoea trifida is belonged to Solanales together with Solanum lycopersicum rather than Arabidopsis thaliana.

Gene structure and conserved domain analysis

The intron/exon structure was determined by analysing genomic DNA with full-length ItfbZIP CDS sequences (Fig. 4). Results indicated that the ItfbZIPs contains at least one intron except ItfbZIP26 with no intron. The D 


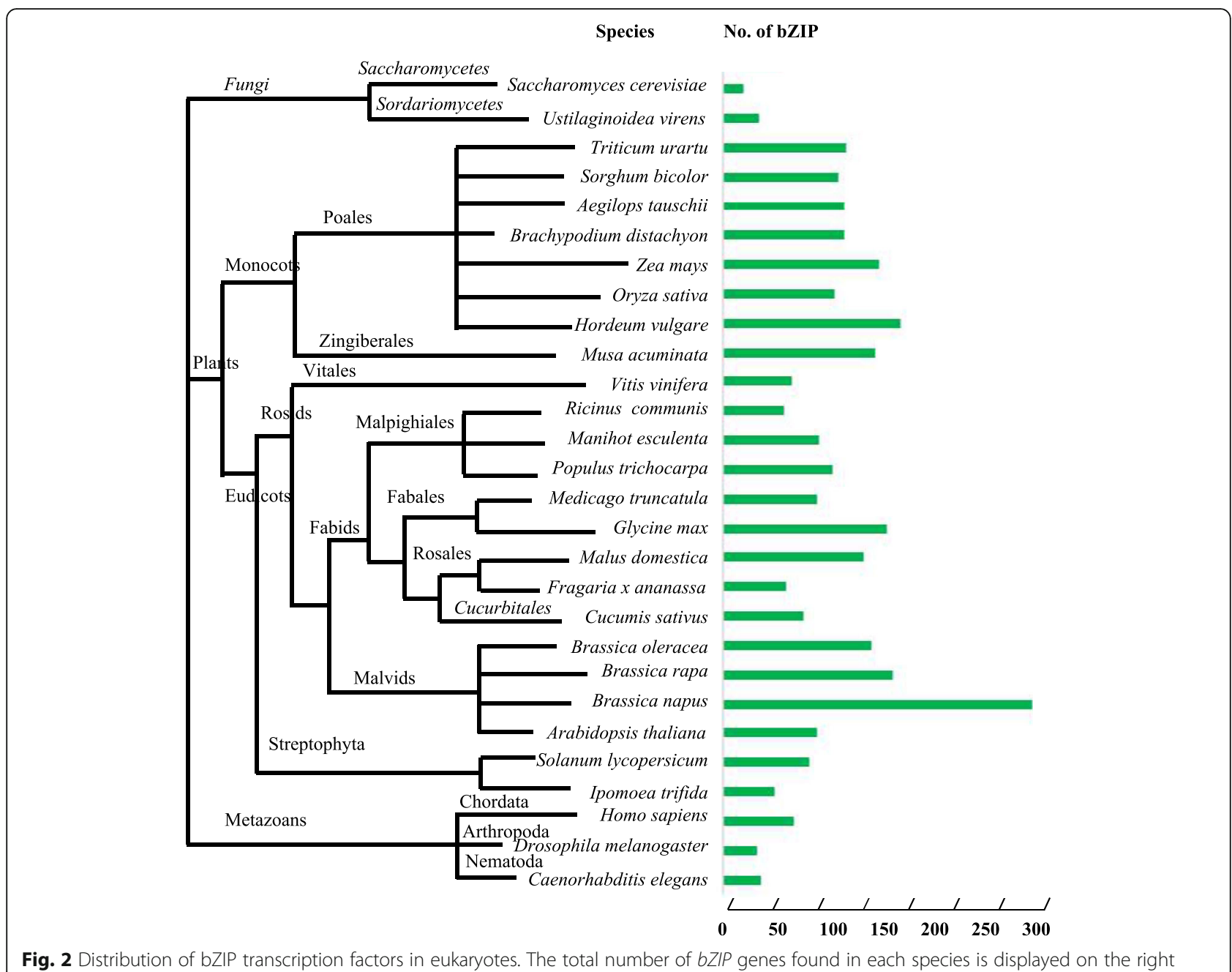

group ItfbZIPs contain 7 to 11 introns, followed by the $G$ group with 4 to 6 introns, the $C$ group with 4 to 5 introns, the $\mathrm{H}$ group with 2 or 3 introns, the $\mathrm{S}$ group with 3 introns, the A, E and I group with 1 to 3 introns, the $\mathrm{B}$ group with 1 intron, and finally the $\mathrm{F}$ group with 0 or 1 intron. The different ItfbZIP transcripts encoded by same ItfbZIP gene have similar exon/intron structure and intron phase patterns.

Conserved domain analysis of the ItfbZIP proteins was performed using the SMART and Pfam databases. The DNA binding ability and the heterozygosity of bZIP TFs are determined by the bZIP conserved domain, which mainly includes the basic domain $(\mathrm{N}-\times 7-\mathrm{R} / \mathrm{K})$ and the leucine zipper domain $(\mathrm{L}-\times 6-\mathrm{L}-\times 6-\mathrm{L})$. The results indicate that the majority of the ItfbZIP family members contain a highly conserved bZIP domain visualized by genedoc software in Additional file 4: Figure S1. In the basic region, only the Asp of five ItfbZIP (ItfbZIP-6, $13,-23.1,-23.2,-29)$ in the E subfamily is replaced by Lys/Gln, and the Arg/Lys of five ItfbZIP (ItfbZIP-25.1, $25.2,-25.3,-25.4,-25.5)$ in the $G$ subfamily are replaced by Ile. Interestingly, the conventional 9 amino acid residues are replaced by 12 amino acid residues at $\mathrm{R} / \mathrm{K}-\times$ 9-L region of ItfbZIP11, which is similar to OsbZIP34 in rice [3]. The leucine zipper region contains a Leu at 7 th amino acid position ( 9 amino acids after $\mathrm{R} / \mathrm{K}$ in the C-terminal extension), but sometimes Leu is replaced by other hydrophobic residues (IIe, Val and Met), such as ItfbZIP12, ItfbZIP13, ItfbZIP23.1, ItfbZIP23.2, ItfbZIP27.1, ItfbZIP27.2.

\section{Cis-element prediction of ItfbZIP genes}

To understand the potential regulatory mechanisms of ItfbZIP gene responding to abiotic stress, cis-elements in the ItfbZIP promoter were analyzed using Analysis Navigator (PlantPAN 2.0) database and plantCARE (Additional file 5: Table S4). Every promoter of ItfbZIP gene has at least one stress related cis-element. Among these elements, $96 \%$ of the ItfbZIP genes contained multiple stress-responsive regulatory elements (HSE stress response element, MBS response element for drought 


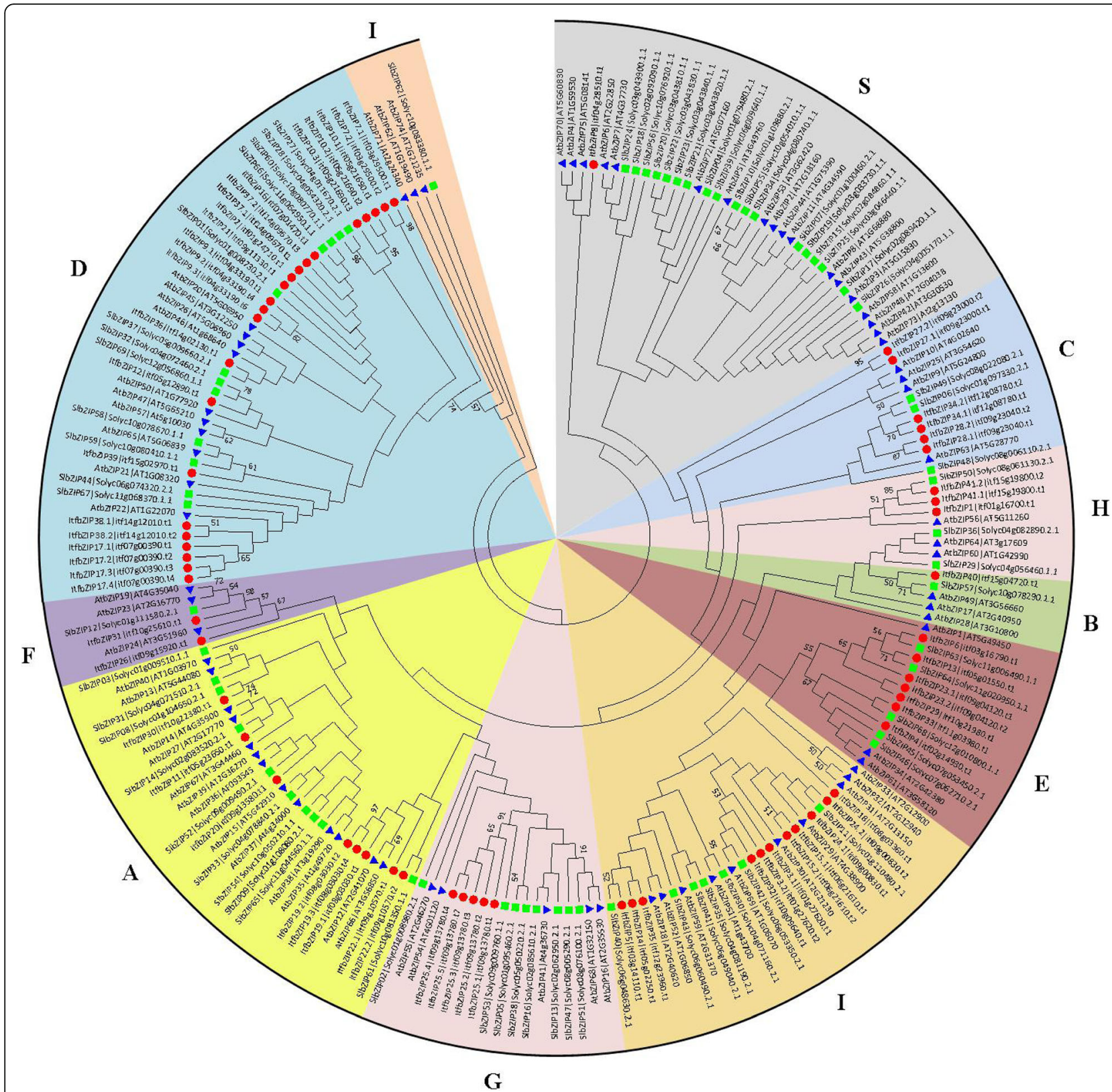

Fig. 3 Phylogenetic relationships among the identified bZIP proteins in Arabidopsis thaliana, Solanum lycopersicum and Ipomoea trifida. The 75 Arabidopsis thaliana, 69 Solanum lycopersicum and 66 Ipomoea trifida bZIP domain protein sequences were aligned by ClustalX, and the phylogenetic tree was constructed using MEGA7 by the Maximum Likelihood method analysis (1000 replicates). Arabidopsis thaliana, Solanum lycopersicum and Ipomoea trifida genes are indicated at the end of the branches. Subgroups A-I and S were named according to Arabidopsis thaliana [6]. The colored regions indicate different subfamilies, the red solid circles indicate the ItfbZIP proteins, the green solid circles represent the SIbZIP proteins, and the blue solid circles represent the AtbZIP proteins

stress, low-temperature stress response element LTR and phosphate starvation responsive element P1BS). This finding indicates that the expression of these ItfbZIP genes is regulated by different abiotic stress. In addition, these ItfbZIP promoters contain hormone response elements (Abscisic acid response element ABRE, gibberellin response element P-box, GARE-motif, TATC-box, auxin response element TGA-box, ethylene response element
ERE, MeJA response element TGCCG-Motif and CGTC A-motif, and salicylic acid responsive element TCA-element). This finding suggests that ItfbZIPs participate in the regulation of plant growth and development and abiotic stress adaption. This result is also consistent with previous cis-acting elemental analyses of the bZIP TFs in Hordeum vulgare L, six fragaria species, Vigna radiata, Vigna angularis and Solanum tuberosum [46-49]. 


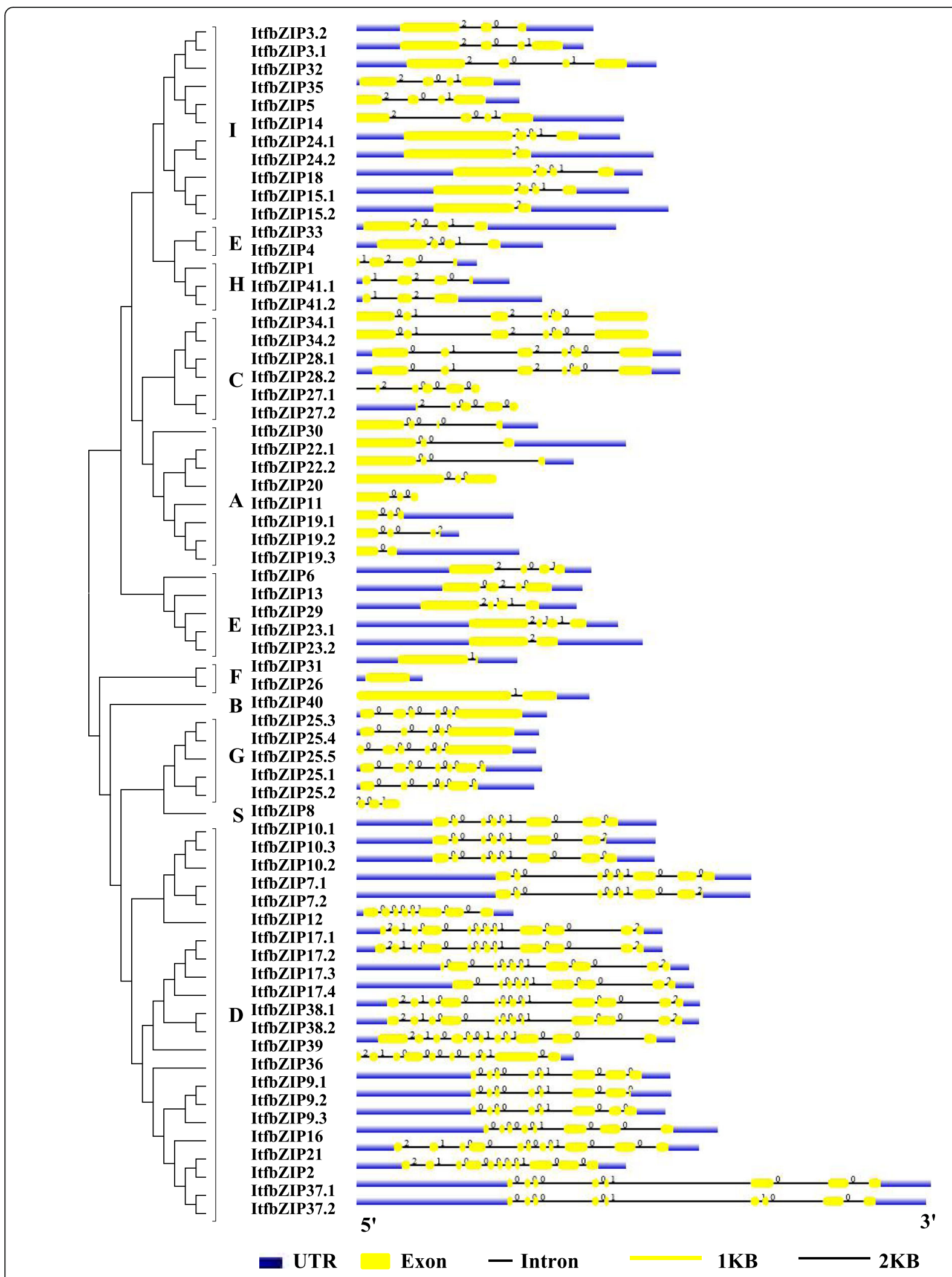

Fig. 4 Exon-intron structures of ItfbZIP genes. The phylogenetic tree on the left was constructed using MEGA 7 software based on the full length sequences of the ItfbZIP proteins.Yellow rectangles represent exons, blue rectangles represent the untranslated regions, black thin lines represent introns 


\section{Interaction network of the ItfbZIP proteins}

Understanding the functional relationships of the ItfbZIP proteins is important for studying the family's regulatory pathways. Thus, we used the STRING software to construct an ItfbZIP gene interaction network based on Arabidopsis thaliana orthologous genes, systematically analysing the interactions of ItfbZIP proteins (Fig. 5). Among the proteins, ABI5 (ItfbZIP20), AREB3 (ItfbZIP-22.1, - 22.1), AT5G44080 (ItfbZIP6), PAN (ItfbZIP36), TGA1 (ItfbZIP-7.2, - 7.1, - 10.1, - 10.2, - 10.3), TGA10 (ItfbZIP39), TGA6 (ItfbZIP-2, - 16, - 21, - 37.1, $-37.2,-9.1,-9.2,-9.3$ ), TGA7 (ItfbZIP12) and TGA9 (ItfbZIP-17.1, - 17.2, - 17.3, - 17.4, - 38.1, - 38.2) are involved in the KEGG signalling pathway of plant hormone signal transduction (ID 4075). TFs that serve other functions were also observed. HY5 (ItfbZIP-1, -41.1 and 41.2) is involved in the downstream reaction of cryptochrome (CRY1 and CRY2) signals. bZIP19 (ItfbZIP31) regulates the expression of zinc transporters ZIP3, ZIP4, ZIP5 and ZIP9 during root growth. BZIP24 and BZIP17
(ItfbZIP40) are involved in salt and osmotic pressure responses. BZIP34 (ItfbZIP8) may play an important role in controlling metabolic pathways that regulate cell transport, and lipid metabolism. BZIP27 (ItfbZIP-11, - 19.2 and -19.3) promotes TFs required for transition to flowering. Therefore, the study of the interactions of the ItfbZIP family members provided us new research ideas for further exploring of new functions of ItfbZIPs.

\section{Homology modeling of ItfbZIP proteins}

Swiss-Model was used to analyze three-dimensional structural homology modeling of ItfbZIP amino acid sequences. Because SWISS-model does not predict effectively for sequences with low homology, we retained 34 models of ItfbZIP proteins with homology higher than $30 \%$. Figure 6 shows the ItfbZIP three-dimensional structural models in the subfamilies A (ItfbZIP-11, - 19.1,-19.2, $-19.3,-20,-22.1,-22.2,-30)$, B (ItfbZIP40), D (ItfbZIP-2, - 7.1, - 7.2, - 10.1, - 10.2, - 10.3, - 12, - 9.1, - 9.2, $-9.3,-16,-17.1,-17.2,-17.3,-17.4,-21,-36,-37$,

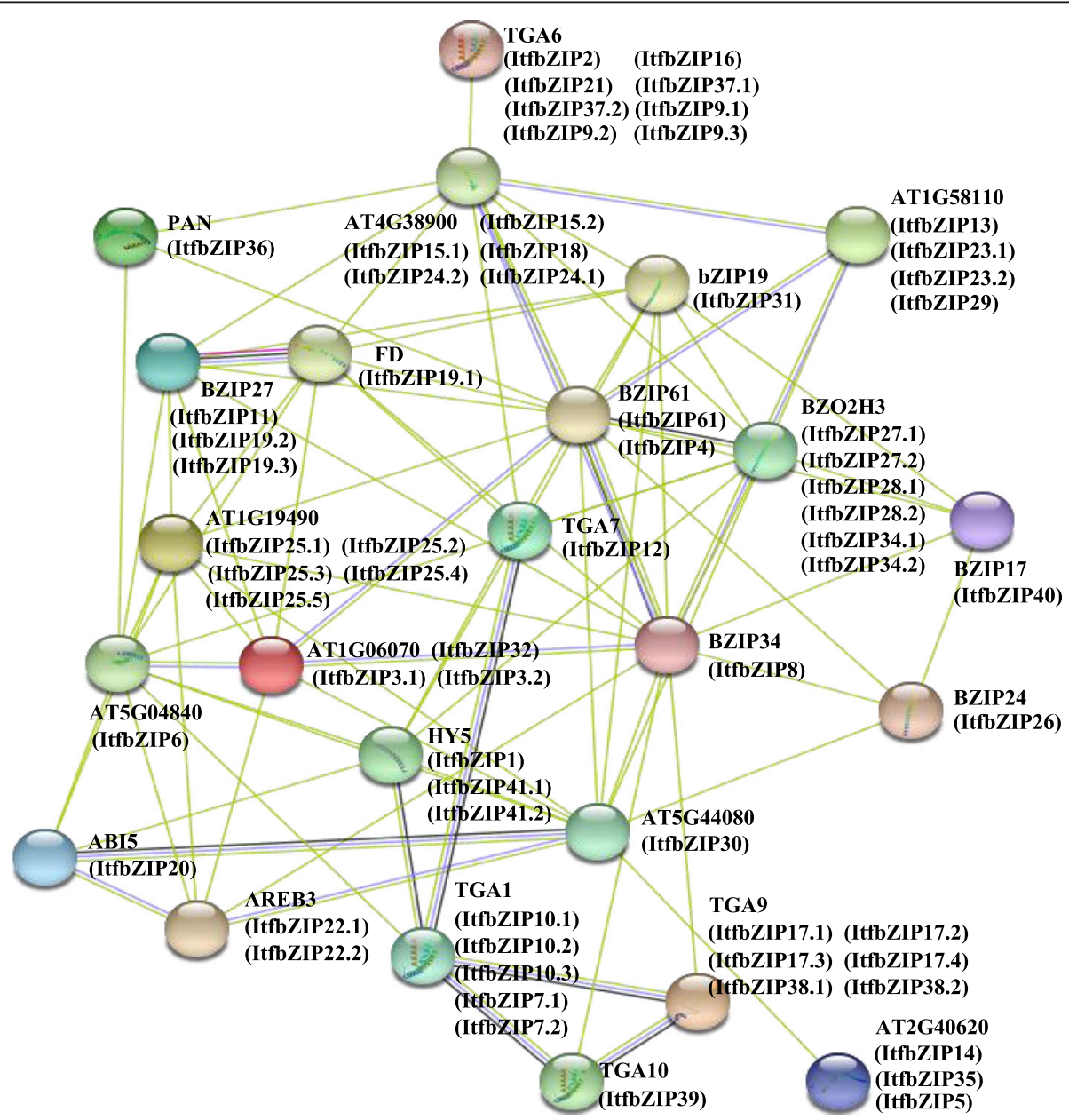

Fig. 5 Functional interaction networks of ItfbZIP proteins in Ipomoea trifida according to orthologues in Arabidopsis thaliana. Network nodes represent proteins, and edges represent protein-protein associations 

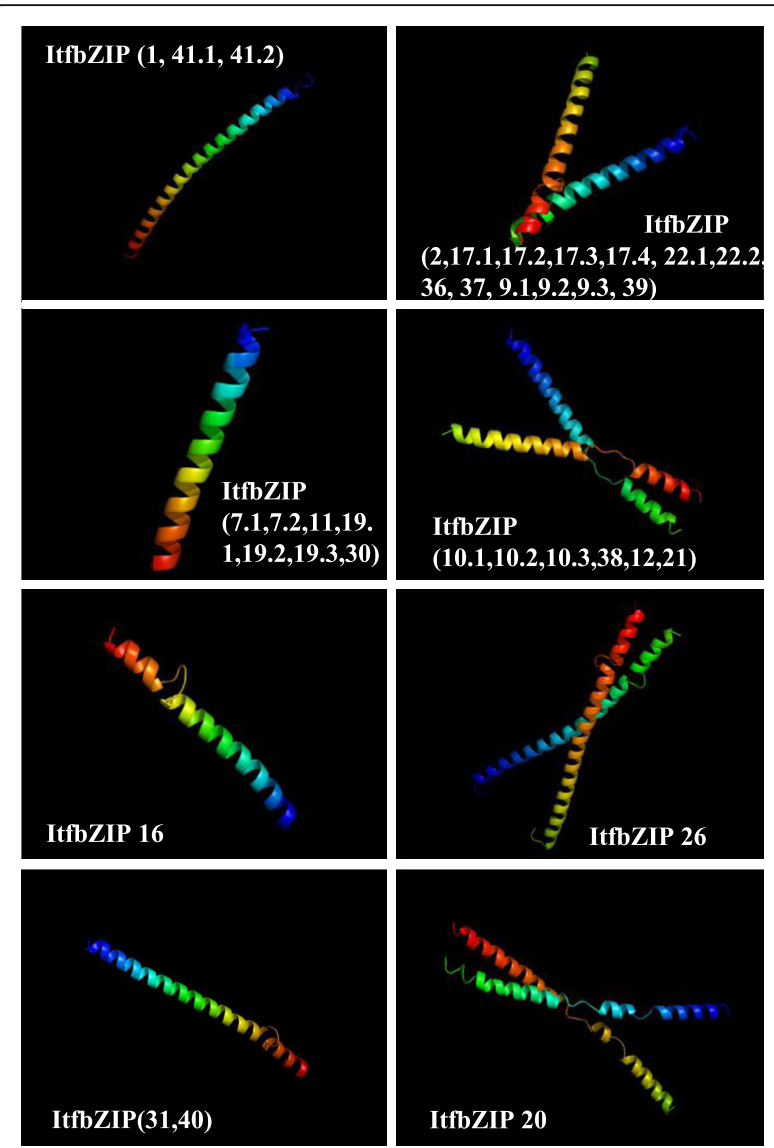

Fig. 6 Homology modeling of ItfbZIP proteins. The figure shows the three-dimensional structural models of 34 ItfbZIP proteins (distributed in the A (ItfbZIP-11, $-19.1,-19.2,-19.3,-20,-22.1,-22.2,-30)$, B (ItfbZIP40), D (ItfbZIP-2, - 7.1, - 7.2, - 10.1, - 10.2, - 10.3, - 12, - 9.1, $-9.2,-9.3,-16,-17.1,-17.2,-17.3,-17.4,-21,-36,-37,-38$, - 39), $F($ (ItfbZIP-26, -31$)$ and $\mathrm{H}$ (ItfbZIP-1, - 41.1, - 41.2) subfamilies) predicted by Swiss-Model software

- 38, - 39), F (ItfbZIP-26, - 31) and H (ItfbZIP-1, - 41.1, -41.2). The results show that all proteins have $\alpha$-helices but no $\beta$-sheets. The ItfbZIPs in $\mathrm{H}$ subfamily mainly contain an $\alpha$-helices. The A, B, D and F subfamily ItfbZIPs contain a few random coiled structures besides $\alpha$-helices. At the same time, the number of $\alpha$-helices and random coils among different members in the same subfamily are different, such as A, D and F subfamily, suggesting that different members in the same subfamily may have different functions.

\section{Gene expression analysis of ItfbZIPs in various tissues of Ipomoea trifida}

To gain insights into the role of the ItfbZIP genes in the growth and development, we used RNA-seq data from seven tissues (callus_flower, callus_stem, flower, flower bud, leaf, root and stem) to study the expression patterns of the ItfbZIP genes in different tissues. As shown in Figs. 7, 40\% of ItfbZIP transcripts share similar expression patterns in the roots, stems, leaves, flower buds and flowers. For example, ItfbZIP2 and ItfbZIP30 were highly expressed in seven tissues, whereas ItfbZIP10.3 and ItfbZIP38.2 were lowly expressed in these tissues. Moreover, the expression patterns of different transcripts of the same gene differ in each tissue. For example, ItfbZIP9.1 was highly expressed in each tissue, whereas ItfbZIP9.2 and ItfbZIP9.3 were lowly expressed. Interestingly, ItfbZIP28.2 was only highly expressed in the leaf. ItfbZIP4 was lowly expressed only in the roots. ItfbZIP17.2 was highly expressed only in the callus_flower and callus_stem. To confirm the expression profiles of ItfbZIP gene obtained from RNA-seq analysis, we randomly selected 14 ItfbZIP genes to investigate their expression in four different tissues (stem, root, leaf and flower) by qRT-PCR (Fig. 8). The results show that the qRT-PCR results matched well with RNA-seq data, such as ItfbZIP2, ItfbZIP6, ItfbZIP10.1, ItfbZIP10.2, ItfbZIP16, ItfbZIP26, ItfbZIP28.1, ItfbZIP31, ItfbZIP32, ItfbZIP41.1.

\section{Expression of ItfbZIP genes under abiotic stress and ABA treatment}

The bZIP TF family plays an important role in plant stress response. Thus, it is very necessarily to investigate the expression of ItfbZIPs under abiotic stress and hormonal treatment. Figure 9 shows the expression of Itfb$Z I P$ genes under drought, salt, cold and heat stress. Among them, the expression of ItfbZIP1, ItfbZIP3.1 and ItfbZIP4O were all up-regulated under these four abiotic stresses. The expression of ItfbZIP10.1, 1tfbZIP3.2, $1 t f b Z I P 21$ and ItfbZIP31 were up-regulated under drought, salt and cold stress. Six genes (ItfbZIP -25.5, $28.1,-18,-6,-17.3$ and -9.2$)$ were up-regulated under heat, salt and drought stress. Only ItfbZIP34.1 was up-regulated under salt, heat and cold stress. The expression of eight ItfbZIP genes (ItfbZIP-25.3, - 25.2, $7.1,-2,-13,-36,-10.2$, and -11 ) were up-regulated under salt and drought stress. Seven ItfbZIP genes (ItfbZIP-4, - 12, - 14, - 15.1, - 7.2, - 17.4, - 35) were all down-regulated under drought, cold, salt and heat stress. To verify the RNA-seq data and further clarify the expression pattern of the ItfbZIPs in detail, gene expression was analyzed by qRT-PCR in plants root, stem and leaf under salt, drought, cold, heat and ABA treatment. Eight ItfbZIP genes (ItfbZIP-1, - 3.1, - 9.1, - 21, - 24.1, $-28.1,-30,-41.1)$ which were up-regulated under at least under one of the four stresses are selected for qRT-PCR analysis (Fig. 10). The results show that the ItfbZIP genes respond to salt, drought, cold, heat or $A B A$ treatment differently. In roots, the gene expression of ItfbZIP1 and ItfbZIP41.1 were up-regulated under drought, cold, heat and ABA stress treatments. And the gene expression of ItfbZIP21 was up-regulated at $12 \mathrm{~h}$ and $24 \mathrm{~h}$ under salt, drought, cold, heat and ABA 


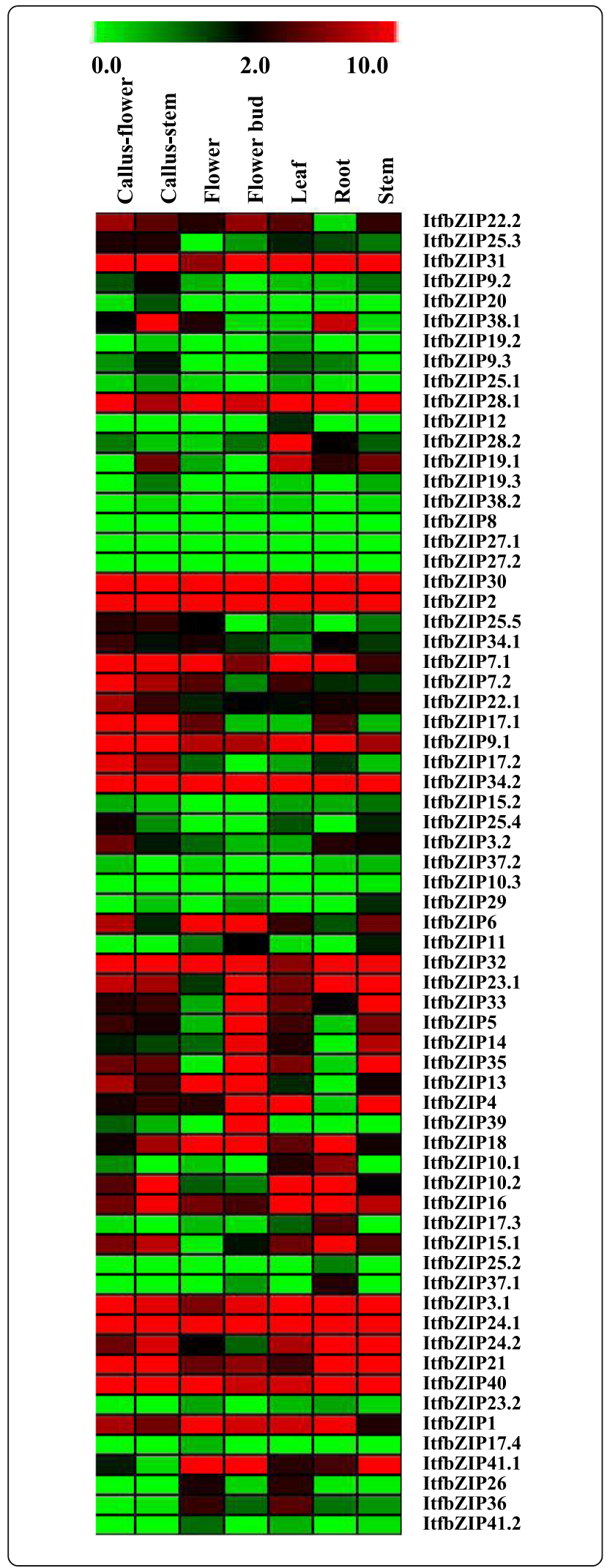

Fig. 7 Relative expression levels of ItfbZIP genes across various tissues. A heat map with clustering is created based on the FPKM value of ItfbZIPs. The coloured scale varies from green to red, indicating relatively low or high expression

stresses. In the stems, ItfbZIP41.1 was up-regulated at $24 \mathrm{~h}$ and $48 \mathrm{~h}$ under these 4 stresses and ABA treatment. In the leaves, the expression of ItfbZIP1 and ItfbZIP30 were up-regulated under drought, cold, heat and $A B A$ stresses. Under ABA treatment, the expression of eight ItfbZIP genes were induced at least in one tissue (root, stem, leaf) and at one time point $(6 \mathrm{~h}, 12 \mathrm{~h}, 24 \mathrm{~h}$ or $48 \mathrm{~h}$ ). The expression of ItfbZIP3.1 was down-regulated at every time point in stem after ABA.

\section{Discussion}

Sweet potato is an important grain, health care and industrial raw material crop that features wide adaptability, high yield and strong resistance to environmental stress. China has the largest planting area and highest yield of sweet potato in the world. However, the genetic background of cultivated sweet potato is complex. The cytogenetics and genomics basis of sweet potato are much weaker than that of other food crops (such as rice, corn and wheat) [50]. However, the genome of Ipomoea trifida as most probably the progenitor of the sweet potato was released recently, which is very helpful for the study of the sweet potato genetic improvement. BZIP is the most widely distributed TF involved in stress resistance in the eukaryote community. In plants, it plays an important role not only in growth and development but also in the response to abiotic and biotic stresses. To date, most of the studies on the physiological and molecular mechanisms of bZIP are focused on Arabidopsis thaliana and rice plants but not on Ipomoea trifida. Whole genome analysis ItfbZIP genes are usually used to screen new varieties of plants with high yield and resistance to stress condition. Genome-wild study of ItfbZIPs in Ipomoea trifida has an important guiding role in the further in-depth study of bZIP gene function and molecular breeding of sweet potato.

\section{Evolution of ItfbZIP genes}

In this study, 41 ItfbZIP genes encoding 66 transcripts were identified. The number of ItfbZIP genes is significantly less than that of other Solanales and cereal crops that have been genome sequenced (Fig. 2), indicating that the ItfbZIP gene family shrinks during the long evolutionary process. All the ItfbZIPs were predicted to be localized in the nucleus; this finding is consistent with the reported experiment results that the CAbZIP1 of pepper, the OsABI5 of rice and the TabZIP1 of wheat are all localized in the nucleus [51-53]. The number of ItfbZIP genes in every chromosome of Ipomoea trifida is 


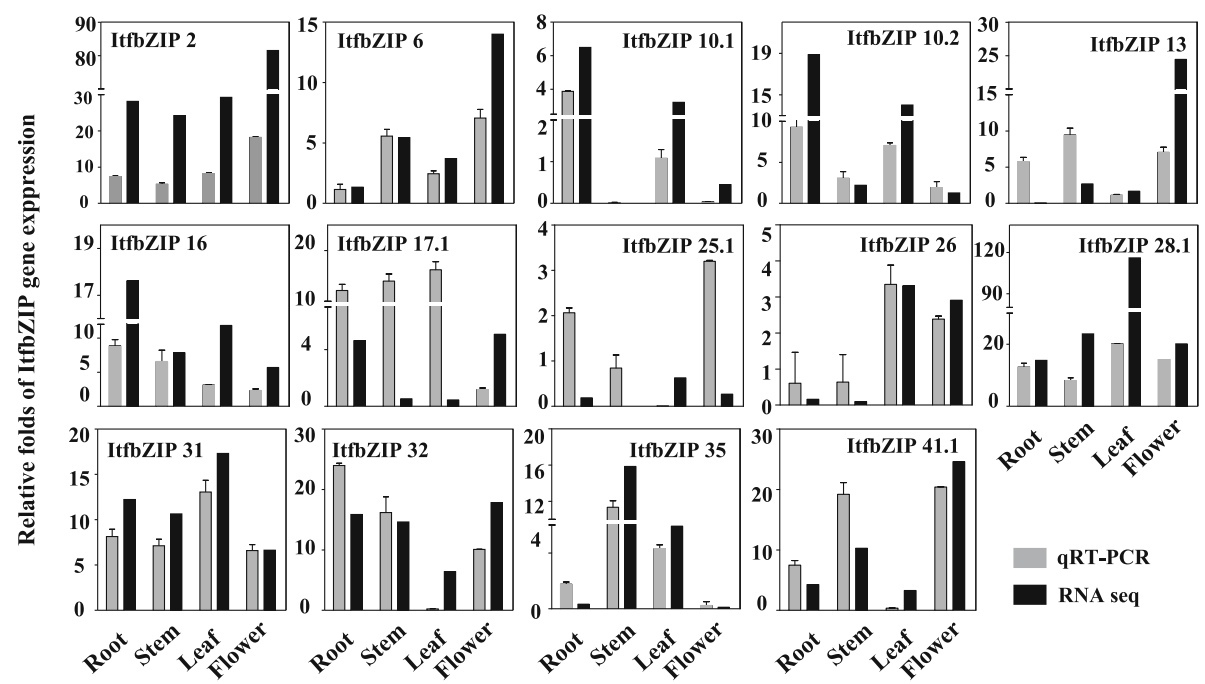

Fig. 8 The comparison between quantitative RT-PCR data and RNA-seq data. The relative expression of the 14 selected ItfbZIP genes was analyzed by qRT-PCR. The GAPDH transcript levels were used for normalization. The $y$-axis represents the relative expression of the fold. Characters on the $x$-axis represent various tissues, Error bars indicate standard deviation. QRT-PCR data represented by gray bars, and black bars represent RNA-seq data

in the range of 1 to 10 (Fig. 1). And ItfbZIPs in Ipomoea trifida showed the similar chromosomal disproportionate distribution pattern with bZIP genes in Hordeum vulgare [49], Cucumis sativus [11] and Medicago truncatula [54]. Segmental and tandem duplication analysis indicates that the contribution of tandem duplication was limited for ItfbZIPs and segmental duplication played a dominant role in the amplification of ItfbZIP gene, which was consistent with the findings in Arabidopsis thaliana, rice and grape [10, 28, 32]. Based on the phylogenetic relationship, ItfbZIP family are divided into ten subfamilies, namely, A, B, C, D, E, F, G, H, I and S (Fig. 3), which is similar to bZIP subfamily classification reported in Maninot esculenta, Musa acuminata and Vitis vinifera. $[10,12,55]$. Moreover, the analysis results of intron/exon gene structure also support phylogenetic analysis. That is, the regularity of the number and distribution of introns is consistent with the subfamily classification from evolutionary tree (Fig. 4). The DNA binding ability and heterogeneity of the bZIP transcription factor are determined by the bZIP domain. Asp (-18), Arg $(-10)$ and Leu $(+1,+8$, $+15)$ are key and invariant sites in the bZIP domain $(\mathrm{N}(-$ 18)- $\times 7-\mathrm{R} / \mathrm{K}(-10)-\times 9-\mathrm{L}(+1)-\times 6-\mathrm{L}(+8)-\times 6-\mathrm{L}(+15)) \quad[9$, 56]. Most of the ItfbZIPs have highly conserved bZIP domains, while a few ItfbZIPs show some substitutions, such as ItfbZIP-6, - $11-13,-23.1,-23.2,-29,-25.1,-25.2$, 25.3, - 25.4, - 25.5 (Fig.S1). Protein homology modeling indicated that $\alpha$-helix is the main structure of ItfbZIP proteins, which is supported by previous research results: the leucine zipper in the conserved region of bZIP forms one amphiphilic $\alpha$-helices, which is the dimerization of bZIP protein before binding to DNA [57].

\section{Expression and potential functions of ItfbZIP genes}

bZIP TFs play essential roles in many biological processes, such as plant growth, development and stress responses et al. In Arabidopsis thaliana, AtbZIP16 can regulate early seedling development by integrating light and hormone signalling pathways and promote seed germination and hypocotyl elongation in the early stage of seedling germination [58]. AtbZIP28 binds to the endoplasmic reticulum membrane and plays an important role in resistance to heat stress [59]. AtbZIP10-LSD1 regulates basic defence and cell death in Arabidopsis thaliana after infection [60]. In rice, OsbZIP12 is a positive regulator of ABA signalling, conferring ABA-dependent drought tolerance [61]. And OsbZIP23 and OsbZIP72 also increase drought resistance through the ABA pathway $[29,62]$. Overexpression of OsbZIP66 and OsbZIP71 enhances the drought tolerance in rice plants [63, 64]. And overexpression of the OsbZIP60 enhances heat and drought resistance [65]. Some bZIP genes have also been studied in Solanales plants. For example, over-expression of $C a H B 1$ gene in tomato can enhance tomato salt tolerance [66]. Expressing tomato SIAREB in Arabidopsis thaliana triggers AtRd29A, AtCOR47 and SICI7-like dehydrin in drought and salt responses [67]. Overexpressing SlAREB1 in tomato can increase the plant tolerance to salt and water stresses [68]. Tobacco bZIP transcription factors TGA2.2 and TGA2.1 have distinct roles in plant defense and plant development [69]. In our study, ItfbZIPs which have high homology with Arabidopsis thaliana and Solanum lycopersicum bZIPs may play similar roles in specific biological processes.

Protein phosphorylation is one of the most important post-translational modifications and plays an essential 


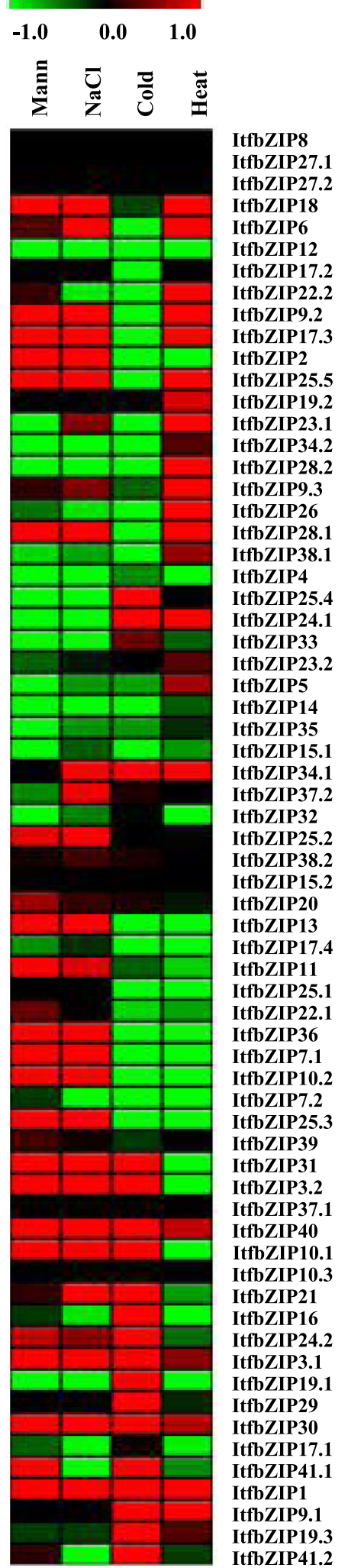

Fig. 9 Expression pattern of ItfbZIP genes under abiotic stress. A heat map created with clusters based on the ItfbZIP FPKM value. Red color indicates a high expression of the relevant gene role in regulating the activity of TFs. Regulating the TF's entry into the nucleus may also change the DNA-binding ability or activity of the TF. The activation of rice TREB protein and Arabidopsis thaliana ABI5 protein require phosphorylation [70, 71]. Activation of threonine/serine casein kinase II (CKII) phosphorylation of G-box-binding factor 1 (GBF1) in Arabidopsis thaliana is associated with plant senescence [72]. bZIP transcription factors AREB1, AREB2 and ABF3 are phosphorylated by SNF1-related protein kinase SRK2D, which activates the cascade response of plants to drought and water stress depending on ABA signaling pathway [36]. The predicted phosphorylation sites of ItfbZIP proteins are shown in Table 1. All ItfbZIP proteins have 4 to 18 phosphorylation sites, suggesting that ItfbZIPs may act through post-transcriptional phosphorylation modification.

bZIP gene is involved in plant tissue and organ development. Investigation of tissue-specific gene expression pattern helps us get some hints about the gene function. RNA-seq data were used to analyse the expression pattern of $b Z I P$ genes in the roots, stems, leaves and flowers of Ipomoea trifida. As shown in Figs. 7, 40\% of the ItfbZIP transcripts have similar expression patterns, and 12 genes are highly expressed in these four tissues. These findings indicate that these genes may play an important role in the plant growth development. Interestingly, ItfbZIP28.2 is highly expressed only in the leaf, ItfbZIP4 is lowly expressed only in the roots and ItfbZIP17.2 is highly expressed only in the callus flower and callus stem, indicating that these genes function in specific organs during the growth and development of Ipomoea trifida.

To date, increasing evidence shows that $b Z I P$ genes are involved in hormonal/abiotic stress and related signal transduction pathways. In A. thaliana, TGA2, TGA5 and TGA6 are essential activators of defence responses induced by salicylic acid (SA)/ethylene [73]. TGA7 is involved in plant drought stress. TGA9 and TGA10 are involved in plant immune responses [74]. Combination analysis of the cis-acting elements and protein interaction networks suggests that ItfbZIPs protein may participate in KEGG signalling pathway and plant hormone signal transduction (ID 4075) (Fig. 5 and Additional file 5: Table S4). In addition, PAN (ItfbZIP36), TGA1 (ItfbZIP-7.2, - 7.1, $10.1,-10.2$ and - 10.3), TGA10 (ItfbZIP39), TGA6 (ItfbZIP-2, - 16, - 21, - 37.1, - 37.2, - 9.1, - 9.2 and -9.3), TGA7 (ItfbZIP12), and TGA9 (ItfbZIP-17.1, - 17.2, 17.3, $-17.4,-38.1$ and -38.2 ) are all Group D ItfbZIPs TFs. And D subfamily genes contain cis-acting elements associated with biotic and abiotic stresses. The gene expression of ItfbZIP7.1 and ItfbZIP36 was up-regulated under drought and salt stresses and down-regulated under cold and heat stresses. The expression of ItfbZIP10.1 was up-regulated under three stresses (droguht, salt and cold) 


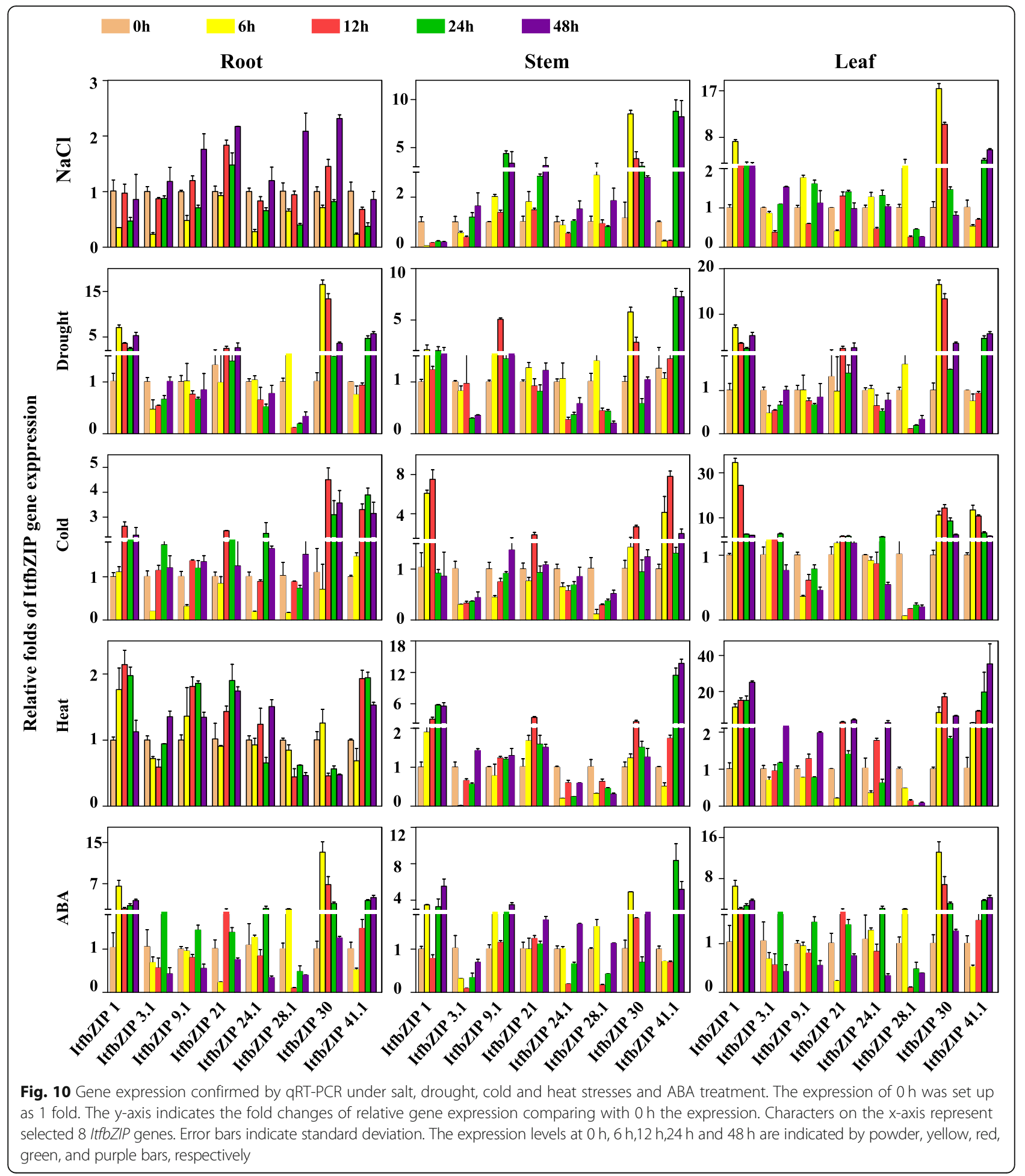

but down-regulated under heat stress. ItfbZIP 17.3 was down-regulated under cold stress and up-regulated under the other three stresses (droguht, salt and heat). This finding suggests Group D ItfbZIPs have specific meaning for abiotic stress response and related signal transduction. The different gene expression patterns of ItfbZIPs indicate
ItfbZIPs play different roles under various abiotic stress conditions (Fig. 9 and Fig. 10).

\section{Conclusion}

Ipomoea trifida genome has 41 ItfbZIP genes. These genes are localized on 14 chromosomes and are 
uniformly named according to their chromosomal location. According to the phylogenetic analysis of Arabidopsis thaliana and Solanum lycopersicum, ItfbZIP gene family is divided into ten subfamilies which have certain genetic relationships and have similar biological functions. The phylogenetic tree can be used to infer the diversity and conservation of genes during evolution. This finding is supported by intron/exon structures, cis-acting elements and protein interaction networks. The expression patterns of the $b Z I P$ gene family members are also analyzed using RNA-Seq data and qRT-RNA. Results reveal that bZIP TFs play an important role in plants abiotic stress responses. This study provides a theoretical basis for the application of ItfbZIP genes in molecular breeding of sweet potato.

\section{Methods}

Plant materials and stress treatments

Ipomoea trifida plants were collected from the Sweet Potato Research Institute, Xuzhou Academy of Agricultural Sciences, National Sweet Potato Industry System, China. The plants were grown in soil and vermiculite (1:1) under the greenhouse conditions of $28 / 22^{\circ} \mathrm{Cday} /$ night and a photoperiod of $16 \mathrm{~h}$ light/ $8 \mathrm{~h}$ dark. And Ipomoea trifida $(2 \mathrm{x})$ were watered every 5 days. In order to analyze different tissues expression profiles of ItfbZIP genes, roots, stems and leaves were sampled from 4-week-old Ipomoea trifida plants and flowers were sampled from 2-month-old Ipomoea trifida plants. To study the expression patterns related various stress and hormone treatments, 4 week old Ipomoea trifida plants were subjected to $200 \mathrm{mM} \mathrm{NaCl}, 300 \mathrm{mM}$ mannitol, 50 uM abscisic acid solution, respectively. For cold and heat treatment, 4 week old Ipomoea trifida plants were subjected to $12{ }^{\circ} \mathrm{C}$ and $40{ }^{\circ} \mathrm{C}$, respectively. Roots, stems and leaves of the above treated plants were sampled after $0 \mathrm{~h}$, $6 \mathrm{~h}, 12 \mathrm{~h}, 24 \mathrm{~h}$ and $48 \mathrm{~h}$ treatments.

\section{Acquisition and identification of $b Z I P$ genes in Ipomoea trifida}

The genome annotations of Ipomoea trifida, Arabidopsis thaliana and Solanum lycopersicum were downloaded from Sweetpotato Genomics Resource (http://sweetpotato.plantbiology.msu.edu/), TAIR (https://www.arabidopsis.org/. jsp) and the Sol Genomics Network (https://solgenomics.net/). Firstly, the candidate bZIP TF members were identified using the Pfam database (http://pfam.xfam.org/search\#tabview=tab1) and HMMER 3.0 software (http://hmmer.janelia.org/). Then, the candidate ItfbZIP members were further confirmed if they contained the bZIP domain via using online programmes NCBI-CDD (https://www.ncbi.nlm.nih. gov/cdd/ Structure/cdd/wrpsb.cgi) and SMART database (http://smart.embl.de/).
Chromosomal distribution and synteny analysis of ItfbZIPs The ItfbZIPs gene were mapped to the Ipomoea trifida chromosome based on the chromosomal location provided in the Sweetpotato Genomics Resource (http://sweetpo tato.plantbiology.msu.edu/). Gene duplication events were analyzed using the Multiple Collinearity Scan toolkit (MCScanX) using default parameters [75]. Finally, the visualization was generated by the circos (version 0.69) (http://circos.ca/) [76].

\section{Protein properties, gene structure and promoter region prediction of ItfbZIP genes}

The molecular weight (MW) and the theoretical isoelectric point $(\mathrm{pI})$ of the ItfbZIP proteins were calculated using the online tool ExPASy (http://e xpasy.org/tools/). The subcellular location of these genes was passed through the software WoLF PSORT (https://wolfpsort. hgc.jp/) forecast. Phosphorylation analysis of the ItfbZIP genes was conducted by the online tool P3DB (http:// www.p3db.org/) [77]. The ItfbZIP genes were graphically displayed on the Ipomoea trifida chromosome. The bZIP gene intron-exon structure map was obtained using the ItfbZIP gene coding sequences and the corresponding genomic sequences together into the GSDS v2.0 (http:// gsds.cbi.pku.edu.cn/) [78]. The Plantpan2.0 (http://plant pan2.itps.ncku.edu.tw/) and plantCARE (http://bioinfor matics.psb.ugent.be/webtools/plantcare/html/) were used to detect cis-elements in the approximately $2000 \mathrm{bp}$ promoter region of each ItfbZIP gene [79].

\section{Phylogenetic tree construction, domain identification and protein homology modeling}

The ClustalX program was used to perform multiple sequence alignments of the bZIPs of Ipomoea trifida, A. thaliana and S. lycopersicum. The Maximum Likelihood method was used to construct the phylogenetic tree by MEGA7 programme [80, 81]. The Bootstrap value was set to 1000 . The sequence alignment of the conserved domain (bZIP domain) of ItfbZIPs was performed by using the online SMART (http://smart.embl-heidelberg.de/) and Pfam database (http://pfam.xfam.org/search\# tabview = tab1), and then visualized using genedoc. STRING software was used to construct functionally interacting protein networks with a confidence parameter set at 0.15 threshold. Online SWISS-MOLD (https://www.swissmodel.expasy. org/) [82] and Pymol software were used for homology modeling of ItfbZIP proteins.

\section{Transcriptome analysis}

The ItfbZIP RNA-seq data were downloaded from the Sweetpotato Genomics Resource (http://sweetpotato.plant biology.msu.edu/). The data are shown in Additional file 6: Tables S5 and Additional file 7: Table S6. The ItfbZIP gene expression levels were calculated as fragments per 
kilobase of exon per million fragments mapped (FPKM). Heat maps of gene expression profiles were drawn using MeV 4.9.0 software.

\section{Quantitative real-time PCR analysis}

RNA prep Pure Kit (Tiangen Biotech, Beijing, China) was used to extract total RNA. First-strand cDNA was synthesized using the PrimescriptTM RT reagent kit (Tsingke, Nanjing, China). And then the reverse transcription product was diluted and used as qRT-PCR template. Glyceraldehyde-3-phosphate Dehydrogenase (GAPDH) gene of Ipomoea trifida was used as internal control to evaluate relative gene expression levels. Primers were designed using online Primer 3 (http://bioinfo.ut.ee/pri mer3-0.4.0/), and all the primers were shown in Additional file 8: Table S7. The experiments were conducted for 3 repetitions and the data were calculated using the $2^{-\triangle \Delta \mathrm{Ct}}$ method [83].

\section{Additional files}

Additional file 1: Table S1. Chromosomal locations and segmental duplication of ItfbZIP genes. (EMF $15058 \mathrm{~kb}$ )

Additional file 2: Table S2. Distribution of bZIP transcription factors in eukaryotes. (XLSX $11 \mathrm{~kb}$ )

Additional file 3: Table S3. Accession numbers of bZIP genes in Ipomoea trifida, Arabidopsis thaliana and Solanum lycopersicum. (XLSX $10 \mathrm{~kb}$ )

Additional file 4: Figure S1. The sequence alignment of the conserved domain (bZIP domain) in ItbZIP transcription factors. The primary structure of the bZIP domain and highly conserved residues of the bZIP domain consensus sequence [N-X7-R/K-X9-L-X6-L-X6-L] are shown at the top of the picture. Vertical bars with different colors on the left show different subfamilies. The black and gray shades in the picture present the same and similar amino acids, respectively. (XLSX $23 \mathrm{~kb}$ )

Additional file 5: Table S4. Cis-elements associated with abiotic stress in promoter region of ItfbZIPS. (XLS $38 \mathrm{~kb}$ )

Additional file 6: Table S5. Relative expression levels of ItfbZIPs in various tissues. (XLSX $30 \mathrm{~kb}$ )

Additional file 7: Table S6. Expression pattern of ItfbZIPs under abiotic stresses. (XLSX $38 \mathrm{~kb}$ )

Additional file 8: Table S7. Primers of the ItfbZIP genes and housekeeping gene for qRT-PCR. (XLSX $10 \mathrm{~kb})$

\section{Abbreviations}

AA: Amino acid; pl, Isoelectric points; ABA: Abscisic acid; bZIP: Basic leucine zipper; ItfbZIP: Basic leucine zipper of Ipomoea trifida; KEGG: Kyoto

Encyclopedia of Genes and Genomes; Leu: Leucine; MW: Molecular weights; TFs: Transcription factors

\section{Acknowledgements}

We thank the GT4SP project team for sharing the Ipomoea trifida genome annotation data (http://sweetpotato.plantbiology.msu.edu/).

\section{Funding}

This work was supported jointly by the projects of the National Natural Science Foundation of China (Grant Nos. 31701481 and 31771367), the Natural Science Foundation of Jiangsu Province (No. BK20160214), the China Agriculture Research System (CARS-10-B03), the Priority Academic Program Development of Jiangsu Higher Education Institutions (No. PAPD) and Jiangsu Overseas Visiting Scholar Program for University Prominent Young \& Middle-aged Teachers and Presidents.
Availability of data and materials

The data sets used and/or analyzed during the current study are available from the corresponding authors on reasonable request.

\section{Author's contributions}

TX and ZL conceived and designed this experiment. ZY, JS, YC and PZ carried out the experiments. DM offered the plant material. ZY and JS analyzed the data. SW, and LZ helped to analyze the data. TX and ZY wrote the manuscript. PZ and QC helped to revise the manuscript. All authors read and approved the manuscript.

\section{Ethics approval and consent to participate}

The authors declare that the experiments of this study comply with the current laws. The data used in the present research were downloaded from the Sweetpotato Genomics Resource (http://sweetpotato.plantbiology.msu. edu/) established by the Michigan State University.

\section{Consent for publication \\ Not applicable.}

\section{Competing interests}

The authors declare they have no competing interests.

\section{Publisher's note}

Springer Nature remains neutral with regard to jurisdictional claims in published maps and institutional affiliations.

\section{Author details}

${ }^{1}$ Institute of Integrative Plant Biology, School of Life Sciences, Jiangsu Normal University, Xuzhou 221116, Jiangsu Province, China. ${ }^{2}$ Key lab of phylogeny and comparative genomics of the Jiangsu province, Jiangsu Normal University, Xuzhou 221116, Jiangsu Province, China. ${ }^{3}$ Department of Plant Biotechnology, College of Agriculture and Life Sciences, Chonnam National University, Gwangju 500-757, South Korea. ${ }^{4}$ Xuzhou Academy of Agricultural Sciences/Sweet Potato Research Institute, CAAS, Xuzhou 221121, Jiangsu, China.

Received: 2 August 2018 Accepted: 4 April 2019

Published online: 25 April 2019

\section{References}

1. Mitchell PJ, Tjian R. Transcriptional regulation in mammalian cells by sequence-specific DNA binding proteins. Science. 1989:245(4916):371.

2. Ptashne M, Gann A. Transcriptional activation by recruitment. Nature. 1997; 386(6625):569.

3. Nijhawan A, Jain M, Tyagi AK, Khurana JP. Genomic survey and gene expression analysis of the basic leucine zipper transcription factor family in rice. Plant Physiol. 2008;146(2):333-50.

4. Paulino PR, Mauricio RPD, Guedes CLG, Rensing SA, Birgit K, Bernd MR. PInTFDB: updated content and new features of the plant transcription factor database. Nucleic Acids Res. 2010;38(Database issue):822-7.

5. Hurst HC. Transcription factors 1: bZIP proteins. Protein Profile. 1994;2(2):123.

6. Jakoby M, Weisshaar B, Drögelaser W, Vicente-Carbajosa J, Tiedemann J, Kroj T, Parcy F. bZIP transcription factors in Arabidopsis. Trends Plant Sci. 2002; 7(3):106-11

7. Liao Y, Zou HF, Wei W, Hao YJ, Tian AG, Huang J, Liu YF, Zhang JS, Chen SY. Soybean GmbZIP44, GmbZIP62 and GmbZIP78 genes function as negative regulator of $A B A$ signaling and confer salt and freezing tolerance in transgenic Arabidopsis. Planta. 2008;228(2):225-40.

8. Wang J, Zhou J, Zhang B, Vanitha J, Ramachandran S, Jiang SY. Genomewide expansion and expression divergence of the basic leucine zipper transcription factors in higher plants with an emphasis on sorghum. J Integr Plant Biol. 2011;53(3):212-31.

9. Wei $K$, Chen J, Wang Y, Chen Y, Chen S, Lin Y, Pan S, Zhong X, Xie D. Genome-wide analysis of bZIP-encoding genes in maize. DNA Res. 2012; 19(6):463-76.

10. Liu J, Chen N, Chen F, Cai B, Santo S D, Tornielli G B, Pezzotti M, Cheng ZM Genome-wide analysis and expression profile of the bZIP transcription factor gene family in grapevine ( Vitis vinifera ). BMC Genomics, 2014, 15(1): $281-281$. 
11. Baloglu MC, Eldem V, Hajyzadeh M, Unver T. Genome-wide analysis of the bZIP transcription factors in cucumber. PLoS One. 2014;9(4):e96014.

12. Hu W, Yang H, Yan Y, Wei Y, Tie W, Ding Z, Zuo J. Genome-wide characterization and analysis of bZIP transcription factor gene family related to abiotic stress in cassava. Sci Rep. 2016;6(22783):22783.

13. Zhao J, Guo R, Guo C, Hou H, Wang X, Hua G. Evolutionary and expression analyses of the apple basic leucine zipper transcription factor family. Front Plant Sci. 2016;7:376.

14. Zhou Y, Xu D, Jia L, Huang X, Ma G, Wang S, Zhu M, Zhang A, Guan M, Lu $\mathrm{K}$, et al. Genome-wide identification and structural analysis of bZIP transcription factor genes in Brassica napus. Genes. 2017;8(10):288.

15. Izawa T, Foster R, Nakajima M, Shimamoto K, Chua NH. The rice bZIP transcriptional activator RITA-1 is highly expressed during seed development. Plant Cell. 1994;6(9):1277-87.

16. Toh S, Mccourt P, Tsuchiya Y. HY5 $\backslash$ r, is involved in strigolactonedependent seed germination in Arabidopsis. Plant Signaling and Behavior. 2012;7(5):556-8.

17. Yin Y. RF2a, a bZIP transcriptional activator of the phloem-specific rice tungro bacilliform virus promoter, functions in vascular development. EMBO J. 1997;16(17):5247-59.

18. Fukazawa J, Sakai T, Ishida S, Yamaguchi I, Kamiya Y, Takahashi Y. Repression of shoot growth, a bZIP transcriptional activator, regulates cell elongation by controlling the level of gibberellins. Plant Cell. 2000;12(6):901-15.

19. Chuang CF, Running MP, Williams RW, Meyerowitz EM. The PERIANTHIA gene encodes a bZIP protein involved in the determination of floral organ number in Arabidopsis thaliana. Genes Dev. 1999;13(3):334-44.

20. Abe M. FD, a bZIP protein mediating signals from the floral pathway integrator FT at the shoot apex. Science. 2005;309(5737):1052-6.

21. Gibalová A, Reňák D, Matczuk K, Dupl'Áková N, Cháb D, Twell D, Honys D. AtbZIP34 is required for Arabidopsis pollen wall patterning and the control of several metabolic pathways in developing pollen. Plant Mol Biol. 2009; 70(5):581-601.

22. Iven T, Strathmann A, Böttner S, Zwafink T, Heinekamp T, Guivarc' HA, Roitsch T, Dröge-Laser W. Homo-and heterodimers of tobacco bZIP proteins counteract as positive or negative regulators of transcription during pollen development. Plant J. 2010;63(1):155-66.

23. Guan YC, Ren HB, He X, Ma ZY, Fan C. Identification and characterization of bZIP-type transcription factors involved in carrot (Daucus carota L.) somatic embryogenesis. Plant J. 2009;60(2):207-17.

24. Ciceri P, Genga A, Viotti A, Schmidt RJ. The activity of the maize opaque2 transcriptional activator is regulated diurnally. Plant Physiol. 1999;121(4):1321-7.

25. Weltmeier F, Ehlert A, Mayer CS, Dietrich K, Wang X, Schütze K, et al. EMBO J. 2006:25(13):3133-43.

26. Baena-González E, Rolland F, Thevelein JM, Sheen J. A central integrator of transcription networks in plant stress and energy signalling. Nature. 2007; 448(7156):938

27. Srivastava R, Howell SH. Stress-induced expression of an activated form of AtbZIP17 provides protection from salt stress in Arabidopsis. Plant Cell and Environment. 2008:31(12):1735-43.

28. Yang O, Popova OV, Süthoff U, Lüking I, Dietz KJ, Golldack D. The Arabidopsis basic leucine zipper transcription factor AtbZIP24 regulates complex transcriptional networks involved in abiotic stress resistance. Gene. 2009:436(1):45-55

29. Lu GJ, Gao CX, Zheng XN, Han B. Identification of OsbZIP72 as a positive regulator of $A B A$ response and drought tolerance in rice. Planta. 2009; 229(3):605-15

30. Hossain MA, Cho J, Han M, Ahn CH, Jeon JS, Park PB, Jungil C, Chulhyun A, Jongseong J, An G. The ABRE-binding bZIP transcription factor OsABF2 is a positive regulator of abiotic stress and $A B A$ signaling in rice. J Plant Physiol. 2010;167(17):1512-20.

31. Hossain MA, Lee $Y$, Cho Jl, Ahn CH, Lee SK, Park PB, An G, Kang H, Lee CH, Jeon JS. The bZIP transcription factor OsABF1 is an ABA responsive element binding factor that enhances abiotic stress signaling in rice. Plant Mol Biol. 2010:72(4-5):557-66

32. Ji X, Liu G, Liu Y, Zheng L, Nie X, Wang Y. The bZIP protein from Tamarix hispida, ThbZIP1, is ACGT elements binding factor that enhances abiotic stress signaling in transgenic Arabidopsis. BMC Plant Biol. 2013;13(1):1-13.

33. Liu C, Wu Y, Wang X. bZIP transcription factor OsbZIP52/RISBZ5: a potential negative regulator of cold and drought stress response in rice. Planta. 2012; 235(6):1157-69.
34. Chen H, Chen W, Zhou J, He H, Chen L, Chen H, Deng XW. Basic leucine zipper transcription factor OsbZIP16 positively regulates drought resistance in rice. Plant Sci. 2012;193-194(3):8-17.

35. Park SH, Jin SJ, Kang HL, Kim YS, Yang DC, Kim JK. OsbZIP23, and OsbZIP45, members of the rice basic leucine zipper transcription factor family, are involved in drought tolerance. Plant Biotechnology Reports. 2015;9(2):89-96.

36. Yoshida T, Fujita Y, Sayama H, Kidokoro S, Maruyama K, Mizoi J, Shinozaki K, Yamaguchi-Shinozaki K. AREB1, AREB2, and ABF3 are master transcription factors that cooperatively regulate $A B R E-d e p e n d e n t ~ A B A$ signaling involved in drought stress tolerance and require ABA for full activation. Plant J. 2010;61(4):14.

37. Shimizu H, Sato K, Berberich T. LIP19, a basic region leucine zipper protein, is a Fos-like molecular switch in the cold signaling of rice plants. Plant Cell Physiol. 2005;46(10):1623-34

38. Rajapakse S, Nilmalgoda SD, Molnar M, Ballard RE, Austin DF, Bohac JR. Phylogenetic relationships of the sweetpotato in Ipomoea series Batatas (Convolvulaceae) based on nuclear beta-amylase gene sequences. Mol Phylogenet Evol. 2004:30(3):623-32.

39. Huang JC, Sun M. Genetic diversity and relationships of sweetpotato and its wild relatives in Ipomoea series Batatas (Convolvulaceae) as revealed by inter-simple sequence repeat (ISSR) and restriction analysis of chloroplast DNA. Theor Appl Genet. 2000;100(7):1050-60.

40. Zhang A, Dai XB, Zhou ZL, Zhao DL, Tang J, Cao QH. Research advance in self-incompatibility of Ipomoea trifida, an ancestor of sweet potato. Acta Agriculturae Jiangxi. 2017;29(5):17-21

41. Lu Y, Sun J, Yang Z, Zhao C, Zhu M, Ma D, Dong T, Zhou Z, Liu M, Yang D, Li Z, Tao X. Genome-wide identification and expression analysis of glycinerich RNA-binding protein family in sweet potato wild relative Ipomoea trifida. Gene. 2019:686:177-86.

42. Cannon S, Mitra A, Baumgarten A, Young N, May G. The roles of segmental and tandem gene duplication in the evolution of large gene families in Arabidopsis thaliana. BMC Plant Biol. 2004;4:10

43. Li Q, Yu H, Cao PB, Fawal N, Mathe C, Azarl S. Explosive tandem and segmental duplications of multigenic families in Eucalyptus grandis. Genome Biol Evol. 2015:7(4):1068-81.

44. Holub EB. The arms race is ancient history in Arabidopsis, the wildflower. Nat Rev Genet. 2001;2(7):516-27.

45. Paterson AH, Bowers JE, Chapman BA. Ancient polyploidization predating divergence of the cereals, and its consequences for comparative genomics. Proc Natl Acad Sci U S A. 2004;101(26):9903-8.

46. Zhao P, Wang D, Wang $\mathrm{R}$, Kong N, Zhang C, Yang C, Wu W, Ma H, Chen Q. Genome-wide analysis of the potato Hsp20 gene family: identification, genomic organization and expression profiles in response to heat stress. BMC Genomics. 2018;19:61.

47. Liu H, Zhong Y, Guo C, Wang XL, Xiong JS, Cheng ZM, Cheng Q. Genomewide analysis and evolution of the bZIP, transcription factor gene family in six fragaria species. Plant Syst Evol. 2017;303:1225-37.

48. Wang L, Zhu J, Li X, Wang S, Wu J. Salt and drought stress and ABA responses related to bZIP genes from $V$. radiata, and $V$. angularis. Gene. 2018:651:152-60.

49. Pourabed E, Golmohamadi FG, Monfared PS, Razavi SM, Shobbar ZS, Soleymani MP. Basic leucine zipper family in barley: genome-wide characterization of members and expression analysis. Mol Biotechnol. 2015;57(1):12-26.

50. Srisuwan S, Sihachakr D, Siljak-Yakovlev S. The origin and evolution of sweet potato (Ipomoea batatas, lam.) and its wild relatives through the cytogenetic approaches. Plant Sci. 2006:171(3):424-33.

51. Lee BJ, Park CJ, Kim SK, Kim KJ, Paek KH. In vivo binding of hot pepper bZIP transcription factor CabZIP1 to the G-box region of pathogenesis-related protein 1 promoter. Biochem Biophys Res Commun. 2006;344(1):55-62.

52. Zou M, Guan $Y$, Ren $H$, Zhang F, Chen F. A bZIP transcription factor, OsABI5, is involved in rice fertility and stress tolerance. Plant Mol Biol. 2008;66(6):675-83.

53. Zhang Y, Zhang G, Xia N, Wang XJ, Huang LL, Kang ZS. Cloning and characterization of a bZIP transcription factor gene in wheat and its expression in response to stripe rust pathogen infection and abiotic stresses. Physiol Mol Plant Pathol. 2008;73(4):88-94.

54. Zhang Z, Liu W, Xiao Q, Liu Z, Xie W, Wang Y. Genome-wide identification, expression profiling, and SSR marker development of the bZIP, transcription factor family in Medicago truncatula. Biochem Syst Ecol. 2015;61:218-28.

55. Hu W, Wang L, Tie W, Yan Y, Ding Z, Liu J, Li M, Peng M, Xu B, Jin Z. Genome-wide analyses of the bZIP family reveal their involvement in the development, ripening and abiotic stress response in banana. Sci Rep. 2016;6:30203 
56. Suckow M, Von WB. B MüllerHill. Identification of three residues in the basic regions of the bZIP proteins GCN4, C/EBP and TAF-1 that are involved in specific DNA binding. EMBO J. 1993;12(3):1193-200.

57. Foley RC, Grossman C, Ellis JG, Llewellyn DJ, Singh KB. Isolation of a maize bZIP protein subfamily: candidates for the ocs-element transcription factor. Plant J. 1993;3(5):669-79.

58. Shen H, Cao K, Wang X. AtbZIP16 and AtbZIP68, two new members of GBFs, can interact with other $\mathrm{G}$ group bZIPs in Arabidopsis thaliana. BMB Rep. 2008;41(2):132-8.

59. Gao H, Brandizzi F, Benning C, Larkin RM. A membrane-tethered transcription factor defines a branch of the heat stress response in Arabidopsis thaliana. Proc Natl Acad Sci U S A. 2008;105(42):16398-403.

60. Kaminaka H, Näke C, Epple P, Dittgen J, Schütze K, Chaban C, Holt BF, Merkle T, Schafer E, Harter K, Dangl J. ZIPI10-LSD1 antagonism modulates basal defense and cell death in Arabidopsis following infection. Plant cell Physiol 47:S43. EMBO J. 2006;25(18):4400-11.

61. Joo J, Lee YH, Song SI. Overexpression of the rice basic leucine zipper transcription factor OsbZIP12 confers drought tolerance to rice and makes seedlings hypersensitive to ABA. Plant Biotechnology Reports. 2014;8(6):431-41.

62. Xiang Y, Tang N, Du H, Ye H, Xiong L. Characterization of OsbZIP23 as a key player of the basic leucine zipper transcription factor family for conferring abscisic acid sensitivity and salinity and drought tolerance in rice. Plant Physiol. 2008;148(4):1938-52.

63. Yoon S, Lee DK, Yu IJ, Kim YS, Yang DC, Kim JK. Overexpression of the OsbZIP66, transcription factor enhances drought tolerance of rice plants. Plant Biotechnology Reports. 2017;11(1):53-62.

64. Liu C, Mao B, Ou S, Wang W, Liu L, Wu Y, Chu C, Wang X. OsbZIP71, a bZIP transcription factor, confers salinity and drought tolerance in rice. Plant Mol Biol. 2014;84(1-2):19.

65. Yu X, Niu X, Yang S, Li Y, Liu L, Tang W, Liu Y. Research on heat and drought tolerance in rice (Oryza sativa L.) by overexpressing transcription factor OsbZIP60. Scientia Agricultura Sinica. 2011:44:4142-49.

66. Oh SK, Yoon J, Choi GJ, Jang HA, Kwon SY, Choi D. Capsicum annuum homeobox 1 (CaHB1) is a nuclear factor that has roles in plant development, salt tolerance, and pathogen defense. Biochem Biophys Res Commun. 2013:442(1-2):116-21.

67. Hsieh TH, Li CW, Su RC, Cheng CP, Sanjaya TYC, Chan MT. A tomato bZIP transcription factor, SIAREB, is involved in water deficit and salt stress responses. Planta. 2010;231(6):1459-73.

68. Orellana S, Yañez M, Espinoza A, Verdugo I, González E, Ruiz-Lara S, Casaretto JA. The transcription factor SIAREB1 confers drought, salt stress tolerance and regulates biotic and abiotic stress-related genes in tomato. Plant Cell and Environment. 2010;33(12):2191-208.

69. Thurow C, Schiermeyer A, Krawczyk S, Butterbrodt T, Nickolov K, Gatz CL. Tobacco bZIP transcription factor TGA2.2 and related factor TGA2.1 have distinct roles in plant defense responses and plant development. Plant J. 2005;44(1):14

70. Hobo T, Kowyama Y, Hattori T. A bZIP factor, TRAB1, interacts with VP1 and mediates abscisic acid-induced transcription. Proc Natl Acad Sci U S A. 1999; 96(26):15348-53.

71. Lynch FTJ. The Arabidopsis abscisic acid response gene ABI5 encodes a basic leucine zipper transcription factor. Plant Cell. 2000;12(4):599-609.

72. Anja S, Stefan F, Ulrike Z. Phosphorylation affects DNA-binding of the senescence-regulating bZIP transcription factor GBF1. Plants. 2015;4(3):691-709.

73. Zander M, La CS, Lamotte O, Métraux JP, Gatz C. Arabidopsis thaliana class-II TGA transcription factors are essential activators of jasmonic acid/ethyleneinduced defense responses. Plant J. 2010;61(2):200-10.

74. Noshi M, Mori D, Tanabe N, Shigeoka S. Arabidopsis clade IV TGA transcription factors, TGA10 and TGA9, are involved in ROS-mediated responses to bacterial PAMP flg22. Plant Sci. 2016;252:12-21.

75. Wang $Y$, Tang H, DeBarry JD, Tan X, Li J, Wang X, Lee TH, Jin H, Marler B, Guo H, Kissinger JC, Paterson AH. MCScanX: a toolkit for detection and evolutionary analysis of gene synteny and collinearity. Nucleic Acids Res, 2012, 40(7):e49-e49.

76. Krzywinski M, Schein J, Birol I, Connors J, Gascoyne R, Horsman D. Circos: an information aesthetic for comparative genomics. Genome Res. 2009;19(9): 1639-45.

77. Yao Q, Bollinger C, Gao J, Xu D, Thelen J J. P3DB: an integrated database for plant protein phosphorylation. Front Plant Sci, 2012, 3: 206-206.

78. Hu B, Jin J, Guo AY, Zhang H, Luo J, Gao G. GSDS 2.0: an upgraded gene feature visualization server. Bioinformatics. 2015;31(8):1296.
79. Higo KY, Iwamoto M, Higo H. PLACE: a database of plant cis-acting regulatory DNA elements. Nucleic Acids Res. 1998;26(1):358-9.

80. Tamura K, Peterson D, Peterson N, Stecher G, Nei M, Kumar S. MEGA5: molecular evolutionary genetics analysis using maximum likelihood, evolutionary distance, and maximum parsimony methods. Mol Biol Evol. 2011;28(10):2731-9.

81. Kumar S, Stecher G, Tamura K. MEGA7: molecular evolutionary genetics analysis version 7.0 for bigger datasets. Molecular Biology and Evolution. 2016;33(7):msw054.

82. Waterhouse A, Bertoni M, Bienert S, Studer G, Tauriello G, Gumienny R, Heer FT, de Beer TAP, Rempfer C, Bordoli L, Lepore R, Schwede T. SWISS-MODEL: homology modelling of protein structures and complexes. Nucleic Acids Res. 2018;46:296-303.

83. Livak KJ, Schmittgen TD. Analysis of relative gene expression data using real-time quantitative PCR and the 2DDCt method. Methods. 2001;25:402-8.
Ready to submit your research? Choose BMC and benefit from:

- fast, convenient online submission

- thorough peer review by experienced researchers in your field

- rapid publication on acceptance

- support for research data, including large and complex data types

- gold Open Access which fosters wider collaboration and increased citations

- maximum visibility for your research: over $100 \mathrm{M}$ website views per year

At BMC, research is always in progress.

Learn more biomedcentral.com/submissions 